Pure and Applied Mathematics Quarterly

Volume 3, Number 1

(Special Issue: In honor of

Robert MacPherson, Part 3 of 3)

107-151, 2007

\title{
Noncommutative Complete Intersections and Matrix Integrals
}

\author{
Pavel Etingof and Victor Ginzburg
}

To Bob MacPherson on the occasion of his 60th birthday

\begin{abstract}
We introduce a class of noncommutatative algebras called representation complete intersections (RCI). A graded associative algebra $A$ is said to be RCI provided there exist arbitrarily large positive integers $n$ such that the scheme $\operatorname{Rep}_{n} A$, of $n$-dimensional representations of $A$, is a complete intersection. We discuss examples of RCI algebras, including those arising from quivers.

There is another interesting class of associative algebras called noncommutative complete intersections (NCCI). We prove that any graded RCI algebra is NCCI. We also obtain explicit formulas for the Hilbert series of each nonvanishing cyclic and Hochschild homology group of an RCI algebra. The proof involves a noncommutative cyclic Koszul complex, $K_{\cdot}^{\mathrm{cyc}} A$, and a matrix integral similar to the one arising in quiver gauge theory.
\end{abstract}

\section{Contents}

1. Introduction

2. Representation functor and matrix integrals

3. Noncommutative complete intersections

4. The trace map

5. Additional results and examples

6. Preprojective algebras and quiver varieties

Received March 11, 2006. 


\section{INTRODUCTION}

1.1. In this paper we work with finitely generated unital associative algebras over $\mathbb{C}$. Such an algebra $F$ is said to be smooth if $\operatorname{Ker}[F \otimes F \rightarrow F]$, the kernel of the multiplication map, is a projective $F$-bimodule.

Fix a smooth algebra $F$, for instance a free algebra $F=\mathbb{C}\left\langle x_{1}, \ldots, x_{d}\right\rangle$. Let $J$ be a two-sided finitely generated ideal in $F$.

Definition 1.1.1. The algebra $A=F / J$ (or the pair $J \subset F$ ) is called a noncommutative complete intersection (NCCI) if $J / J^{2}$ is a projective A-bimodule. This is equivalent to the condition that $A$ has a projective $A$-bimodule resolution of length $\leq 2$, cf. Theorem 3.1.1.

NCCI algebras have been considered, under various different names, by a number of authors, see e.g. [AH], [An], [GS], [Go], [Pi]. In the present paper, we are interested in the case of graded NCCI algebras. Specifically, fix a finite set $I$ and write $R$ for the algebra of functions $I \rightarrow \mathbb{C}$, with pointwise multiplication. Let $Q$ be a finite quiver with vertex set $I$, and assign to each edge $a \in Q$ an arbitrary positive grade degree $d(a)>0$. This makes the path algebra of $Q$ a free graded $R$-algebra; moreover, any free graded $R$-algebra $F$ may be obtained in this way.

We study algebras of the form $A=F / J$, where $J$ is a graded two-sided ideal in the path algebra $F$ such that $J / J^{2}$ is a projective finitely generated $A$-bimodule. Thus, $A=\bigoplus_{r \geq 0} A[r]$ is a graded algebra such that $A[0]=R$.

The grading on $A$ induces a natural grading on each Hochschild homology group $H_{k}(A)$. Our main results (Theorem 2.2.7 and Theorem 3.7.7) give explicit formulas for the Hilbert series of each Hochschild homology group of $A$ for a class of algebras $A$ that we call asymptotic representation complete intersections (see below).

For $k=0$, for instance, Hochschild homology reduces to the commutator quotient space, $H H_{0}(A)=A /[A, A]$, where $[A, A]$ denotes the $\mathbb{C}$-linear subspace of $A$ spanned by the commutators $a b-b a, a, b \in A$. The grading on $A$ clearly descends to the commutator quotient. Our result yields a formula, in terms of an infinite product of determinants, for the Hilbert series of the graded algebra

$$
\mathcal{O}(A)=\operatorname{Sym}_{\mathbb{C}}\left(A_{+} /[A, A]\right) .
$$

Here, $A_{+}=\bigoplus_{r>0} A[r]$, so $A_{+} /[A, A]$ is the positive degree part of $A /[A, A]$. We remark that the symmetric algebra in (1.1.2) is equipped with the total grading that comes from the grading on $A_{+} /[A, A]$ (this grading should not be confused with the standard grading Sym $=\bigoplus_{k \geq 0} \mathrm{Sym}^{k}$, on the Symmetric algebra of a vector space). 
1.2. The main idea of our approach is based on the notion of representation functor and may be outlined as follows.

Let $A$ be any graded finitely presented algebra. For each positive dimension $\mathbf{d}$, one has an affine scheme $\operatorname{Rep}_{\mathbf{d}} A$ of $\mathbf{d}$-dimensional representations of $A$, cf. $\S 2.1$. Heuristically, one expects that the collection of representation schemes $\operatorname{Rep}_{\mathbf{d}} A$ provides, as $\mathbf{d} \rightarrow \infty$, a 'good approximation' of the algebra $A$ (this is not true for nongraded algebras which may have no finite dimensional representations at all). In particular, one should be able to read off a lot of information about $A$ from some geometric information about the corresponding representation schemes.

In more detail, for each $\mathbf{d}$, the general linear group $G L_{\mathbf{d}}$ acts naturally on the scheme $\operatorname{Rep}_{\mathbf{d}} A$ by base change transformations of the underlying vector space of the d-dimensional representation. We consider the algebra $\mathbb{C}\left[\operatorname{Rep}_{\mathbf{d}} A\right]^{G L_{\mathbf{d}}}$, of $G L_{\mathbf{d}}$-invariant polynomial functions on $\operatorname{Rep}_{\mathbf{d}} A$. We show that, in some sense, the commutative algebra $\mathcal{O}(A)$, in (1.1.2), is a limit of the algebras $\mathbb{C}\left[\operatorname{Rep}_{\mathbf{d}} A\right]^{G L_{\mathbf{d}}}$ as $\mathbf{d}$ goes to infinity. A precise version of this statement will be proved in $\S 4$. The statement implies, in particular, that the Hilbert series of $\mathcal{O}(A)$ may be obtained as a limit of Hilbert series of the algebras $\mathbb{C}\left[\operatorname{Rep}_{\mathbf{d}} A\right]^{G L_{\mathbf{d}}}$ as $\mathbf{d}$ goes to infinity.

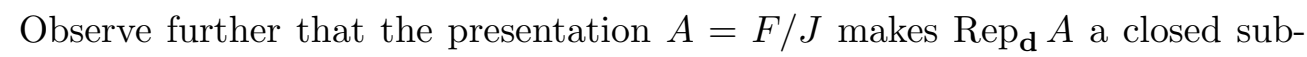
scheme in $\operatorname{Rep}_{\mathbf{d}} F$, the latter being a vector space. We introduce a noncommutative cyclic Koszul complex, $K_{\bullet}^{\text {cyc }} A$, such that each of the functors $\operatorname{Rep}_{\mathbf{d}}$, $\mathbf{d} \in \mathbb{Z}^{I}$, sends $K_{\cdot}^{\text {cyc }} A$ to the $G L_{\mathbf{d}}$-fixed part of the standard Koszul complex of the subscheme $\operatorname{Rep}_{\mathbf{d}} A \subset \operatorname{Rep}_{\mathbf{d}} F$. Moreover, we prove that the two complexes become 'asymptotically isomorphic' as $\mathbf{d} \rightarrow \infty$.

To be able to exploit usefully the 'asymptotic isomorphisms' above we introduce the notion of representation complete intersection (RCI) algebra, and a weaker (but more useful) notion of asymptotic RCI algebra. The algebra $A=F / J$ is called RCI provided there exist arbitrarily large positive dimensions $\mathbf{d}$ such that the scheme $\operatorname{Rep}_{\mathbf{d}} A$ is a complete intersection in $\operatorname{Rep}_{\mathbf{d}} F$. We will prove:

RCI $\Rightarrow$ asymptotic RCI $\Leftrightarrow$ NCCI with 'expected' $H H_{2}(A) \Rightarrow$ NCCI.

It turns out that, for an asymptotic RCI algebra $A$, there is enough asymptotic information about Koszul complexes of the schemes $\operatorname{Rep}_{\mathbf{d}} A$ to deduce formulas for the Hilbert series of each Hochschild homology group of $A$.

1.3. Quiver algebras. Our results apply, in particular, in an interesting special case arising from quivers. Specifically, let $Q$ be a (nonoriented) graph with vertex set $I$. Let $\bar{Q}$ be the quiver obtained by doubling edges of $Q$ so that each nonoriented edge of $Q$ gives rise to a pair, $a, a^{*}$, of oriented edges of $\bar{Q}$ equipped 
with opposite orientations. Write $\mathbf{c}=\left\|\mathbf{c}_{i j}\right\|$ for the adjacency matrix of $\bar{Q}$, thus $\mathbf{c}_{i j}$ denotes the number of edges $i \rightarrow j$ in $\bar{Q}$.

We take $F=F(Q)$ to be the path algebra of $\bar{Q}$. Let

$$
\Pi=F(Q) /\left(\sum_{a \in Q}\left[a, a^{*}\right]\right)
$$

be the preprojective algebra associated to $Q$, a quotient of $F(Q)$ by the two-sided ideal generated by the element $\sum_{a \in Q}\left(a \cdot a^{*}-a^{*} \cdot a\right) \in F(Q)$. The grading on the path algebra induces natural gradings on $\Pi$ and on $\Pi /[\Pi, \Pi]$.

Write $\mathbf{1}$ for the identity $I \times I$-matrix. Thus, $\mathbf{1}-t \cdot \mathbf{c}+t^{2} \cdot \mathbf{1}$ is an $I \times I$-matrix with entries in $\mathbb{Z}[t]$. This matrix specializes at $t=1$ to the Cartan matrix of $Q$. The closely related matrix $\frac{1}{t}\left(\mathbf{1}-t \cdot \mathbf{c}+t^{2} \cdot \mathbf{1}\right)=\left(t+t^{-1}\right) \cdot \mathbf{1}-\mathbf{c}$, called $t$-analogue of the Cartan matrix, has been considered by Kostant and Lusztig, see [Lu, §3.12-3.13].

Applying our general theorem to the algebra $\Pi$ yields the following result.

Theorem 1.3.1. Let $Q$ be neither Dynkin nor extended Dynkin quiver. Then, $\Pi=\Pi(Q)$ is an RCI algebra, and the Hilbert series of $\Pi$, resp. of $\Pi /[\Pi, \Pi]$, is determined from the formula

$h(\Pi ; t)=\left(\mathbf{1}-t \cdot \mathbf{c}+t^{2} \cdot \mathbf{1}\right)^{-1}, \quad$ resp. $\quad h(\mathcal{O}(\Pi) ; t)=\frac{1}{1-t^{2}} \prod_{r \geq 1} \frac{1}{\operatorname{det}\left(\mathbf{1}-t^{r} \cdot \mathbf{c}+t^{2 r} \cdot \mathbf{1}\right)}$.

Here, the formula for $h(\Pi, t)$ has been known for some time, see e.g. [MOV] and references therein, while the formula for $h(\mathcal{O}(\Pi) ; t)$ is new.

Recall that, according to Van den Bergh [VdB], there are natural isomorphisms $H H .(\Pi) \cong H H^{2-\cdot}(\Pi)$ relating Hochschild homology and cohomology. These isomorphisms and the exact sequence (2.2.8), see Theorem 2.2.7 below, yield formulas for Hilbert series of all Hochschild cohomology groups of the algebra $\Pi$. In particular, this gives an alternate proof of the result that, for any quiver of neither Dynkin nor extended Dynkin type, the center of the algebra $\Pi$ reduces to scalars, cf. [CBEG, Proposition 8.2.2].

Both formulas of Theorem 1.3.1 fail for $A, D, E$ Dynkin quivers.

Now, let $Q$ be an extended Dynkin quiver with extending vertex $o \in I$. Then, the formula for $h(\Pi ; t)$ given in Theorem 1.3.1 still holds, but the formula for $h(\mathcal{O}(\Pi) ; t)$ has to be modified, see Corollary 6.3.3 in $\S 6.3$, and [Su]. In particular, that modified formula implies a curious identity for the adjacency matrix of $\bar{Q}$ which we were unable to find in the literature.

The identity involves Chebyshev polynomials of the first kind. Specifically, put $\varphi_{0}:=1$ and let $\varphi_{k} \in \mathbb{C}[x], k=1,2, \ldots$, be a sequence of polynomials in an indeterminate $x$ defined as the coefficients in the expansion of the generating 
function

$$
\frac{2-t x}{1-t x+t^{2}}=1+\sum_{k=1}^{\infty} \varphi_{k}(x) t^{k}, \quad \text { thus } \quad \varphi_{k}(2 \cos z):=2 \cos (k z), \quad \forall k \geq 1
$$

For each $k \geq 0$, we may plug the adjacency matrix $\mathbf{c}$, of $\bar{Q}$, into the polynomial $\varphi_{k}$. This way, we obtain an integer valued $I \times I$-matrix $\varphi_{k}(\mathbf{c})=\left\|\varphi_{k}(\mathbf{c})_{i j}\right\|$. The matrix entries corresponding to the extended vertex $i=j=o$ form a sequence of integers $\varphi_{k}(\mathbf{c})_{o o}, k=0,1, \ldots$ Our identity reads

$$
\prod_{r \geq 1} \operatorname{det}\left(\mathbf{1}-t^{r} \cdot \mathbf{c}+t^{2 r} \cdot \mathbf{1}\right)=\prod_{k \geq 1}\left(1-t^{k}\right)^{\varphi_{k}(\mathbf{c})_{o o} .}
$$

This identity will be proved in $\S 6.3$ by applying the Euler-Poincaré principle to $K^{\text {cyc }} \Pi$, the noncommutative cyclic Koszul complex of the preprojective algebra $\Pi$. One can also verify the identity case by case, for each extended Dynkin quiver of $A, D, E$ types separately, see $\S 6.3$ for details.

1.4. Layout of the paper. In Section 2 , we formulate our first important result (Theorem 2.2.7) and carry out crucial matrix integral calculations. The notion of RCI algebra is introduced in $\S 2.2$ and the notion of asymptotic RCI algebra is introduced in $\S 2.4$. We use the standard Koszul complex associated with a complete intersection to express the Hilbert series of the coordinate ring $\mathbb{C}\left[\operatorname{Rep}_{\mathbf{d}} A\right]$ in terms of a matrix integral, an integral over the unitary group $U_{\mathbf{d}}$. Then, in $\S 2.4$, we apply some results from the theory of random matrices to find the asymptotics of our matrix integral as $\mathbf{d} \rightarrow \infty$. For an asymptotic RCI algebra, that enables us to obtain an asymptotic formula for the Hilbert series of the algebras $\mathbb{C}\left[\operatorname{Rep}_{\mathbf{d}} A\right]$ as $\mathbf{d}$ goes to infinity.

In $\S 3$ we discuss NCCI algebras and related homological algebra. Similarly to the familiar case of commutative algebras, to any noncommutative algebra $A$ given by generators and relations one can associate canonically a free DG algebra $K . A$, called the (noncommutative) Koszul complex of $A$. The noncommutative Koszul complex is defined in $\S 3.2$. It turns out that $A$ is an NCCI algebra if and only if the DG algebra $K . A$ is quasi-isomorphic to $A$. Main results about NCCI algebras are gathered in Theorems 3.1.1 and 3.2.4.

In $\S 3.7$, we introduce the cyclic Koszul complex $K_{\bullet}^{\text {cyc }} A$ that computes cyclic homology of the NCCI algebra $A$. We prove that these vanish in all degrees $>1$. We formulate the main result of the paper, Theorem 3.7.7, which claims, among other things, the equivalence in the middle of (1.2.1).

The proofs of the two main theorems are completed in $\S 4$. In that section, we compare the algebras $A$ and $\mathbb{C}\left[\operatorname{Rep}_{\mathbf{d}} A\right]$ via natural evaluation maps. In particular, we construct an evaluation morphism from the cyclic Koszul complex $K_{\bullet}^{\mathrm{cyc}} A$ to 
the ordinary Koszul complex of the representation scheme $\operatorname{Rep}_{\mathbf{d}} A$, that commutes with the respective Koszul differentials. We prove that, in the $\mathbf{d} \rightarrow \infty$ limit, evaluation morphisms become isomorphisms. Thus, homology vanishing for the noncommutative Koszul complex $K^{\text {cyc }} A$ is equivalent to an asymptotic homology vanishing for the ordinary Koszul complexes of representation schemes $\operatorname{Rep}_{\mathrm{d}} A$. Furthermore the Hilbert series of $K_{0}^{\mathrm{cyc}} A$ may be expressed in terms of Hilbert series of the corresponding Koszul complexes of representation schemes.

In $\S 5$, we consider various examples of RCI and asymptotic RCI algebras. We also provide examples of non RCI algebras which, nevertheless, are asymptotic RCI, see $\S 5.4$. Specifically, we show that if the relations of $A$ are pairwise nonoverlapping monomials, then $A$ is an asymptotic RCI but not necessarily RCI. We use a deformation argument to deduce a similar result for algebras with generic homogeneous relations of degrees equal to the lengths of these non-overlapping monomials. This way, one obtains many nontrivial examples of asymptotic RCI algebras with explicit infinite product formulas for the corresponding Hilbert series.

In $\S 6$, we show that, for connected quivers of neither Dynkin nor extended Dynkin type, the corresponding preprojective algebra is RCI. Also, partial preprojective algebras of any connected quiver (in the sense of [EE]), are RCI algebras. In $\S 6.2$, we give an interesting asymptotic formula for Hilbert series of Nakajima quiver varieties. Extended Dynkin quivers are discussed in $\S 6.3$, where we prove the identity in (1.3.3).

Remark 1.4.1. In the present paper, the ground field is the field $\mathbb{C}$ of complex numbers. All the results of the paper can be routinely generalized to the setting of an arbitrary algebraically closed ground field of characteristic zero. Characteristic zero assumption is essential for the geometric results involving representation schemes. It is also essential for the cyclic/Hochschild homology computation in Lemma 3.6.1 and Proposition 3.7.1, which involves Karoubi-de Rham complex. However, the rest of $\S 3$ applies verbatim in the case of ground fields of arbitrary characteristic.

1.5. Ackmowledgments. The authors are grateful to M. Artin, L. Hesselholt, G. Lusztig, H. Nakajima, D. Piontkovsky, E. Rains, and R. Stanley for useful comments and discussions. We also thank E. Rains for explaining to us the results of Diaconis and Shahshahani on matrix integrals, and for providing a MAGMA code that we used at all stages of this work. The work of P.E. and V.G. was partially supported by the NSF grants DMS-0504847 and DMS-0303465, respectively, and by the CRDF grant RM1-2545-MO-03.

\section{REPRESENTATION FUNCTOR AND MATRIX INTEGRALS}


2.1. Basic definitions. Throughout, we fix a finite set $I$ and let $R$ be the algebra of functions $I \rightarrow \mathbb{C}$, with pointwise multiplication. Thus, $R$ is a commutative semisimple $\mathbb{C}$-algebra. Simple $R$-modules are 1-dimensional and are parametrized by elements of the set $I$; an $R$-module is the same thing as an $I$-graded vector space, $M=\oplus_{i \in I} M_{i}$. If $M$ is finite-dimensional and $d_{i}=\operatorname{dim} M_{i}$, we say that $\mathbf{d}=\left\{d_{i}\right\}_{i \in I}$, an $I$-tuple of nonnegative integers, is the dimension vector for $M$.

Similarly, $R$-bimodules are $I \times I$-graded vector spaces, $M=\oplus_{i, j \in I} M_{i j}$.

Let $M=\bigoplus_{r>0} M[r]$ be a $\mathbb{Z}_{+}$-graded $R$-bimodule such that each graded component $M[r]$ is a finite dimensional $R$-bimodule, that is we have $M[r]=$ $\bigoplus_{i, j \in I} M_{i j}[r]$, where $\operatorname{dim} M_{i j}[r]<\infty$. The Hilbert series of $M$ is a $\mathbb{Z}[[t]]$-valued $I \times I$-matrix defined as

$$
h(M)=\left\|h_{i j}(M ; t)\right\|, \quad h_{i j}(M ; t):=\sum_{r \geq 0} \operatorname{dim} M_{i j}[r] \cdot t^{r} .
$$

It is clear that $h\left(M \otimes_{R} M^{\prime}\right)=h(M) \cdot h\left(M^{\prime}\right)$ for any $\mathbb{Z}_{+}$-graded $R$-bimodules $M$ and $M^{\prime}$.

Given a dimension vector $\mathbf{d}$, let $\mathbb{C}^{\mathbf{d}}:=\bigoplus_{i \in I} \mathbb{C}^{d_{i}}$. This is an $I$-graded vector space, and we consider an associative algebra

$$
\mathbf{E}_{\mathbf{d}}:=\operatorname{Hom}_{\mathbb{C}}\left(\mathbb{C}^{\mathbf{d}}, \mathbb{C}^{\mathbf{d}}\right)=\bigoplus_{i, j \in I} \mathbf{E}_{i j}, \quad \text { where } \quad \mathbf{E}_{i j}=\operatorname{Hom}_{\mathbb{C}}\left(\mathbb{C}^{d_{i}}, \mathbb{C}^{d_{j}}\right) .
$$

The $I$-grading makes the vector space $\mathbb{C}^{\mathbf{d}}$ a left $R$-module, and the action map for this module gives a natural algebra homomorphism $R \rightarrow \bigoplus_{i \in I} \mathbf{E}_{i i} \subset \mathbf{E}_{\mathbf{d}}$.

Notation 2.1.1. We use unadorned symbols like Sym, $\Lambda, \mathrm{Hom}, \otimes$, etc., for symmetric algebra, exterior algebra, Hom-space, tensor product, etc., all taken over $\mathbb{C}($ not over $R$ ).

Given a finite dimensional $R$-bimodule $M$, we reserve the corresponding blackboard font notation for the vector space

$$
\mathbb{M}_{\mathbf{d}}:=\operatorname{Hom}_{R-\operatorname{bimod}}\left(M, \mathbf{E}_{\mathbf{d}}\right) .
$$

The exceptions to this convention are: $\mathbb{N}, \mathbb{Z}_{+}, \mathbb{Z}$, and $\mathbb{C}$, the sets of natural numbers, nonnegative integers, integers, and complex numbers, respectively.

An $\mathbb{N}$-grading on an $R$-bimodule $M$ gives rise to a linear $\mathbb{C}^{\times}$-action on the vector space $\mathbb{M}_{\mathbf{d}}$. This action contracts the vector space to its origin, the only $\mathbb{C}^{\times}$-fixed point.

Let $F$ be an $R$-algebra, that is, a finitely presented associative unital $\mathbb{C}$-algebra equipped with an algebra imbedding $R \hookrightarrow F$. Given a dimension vector $\mathbf{d}$, let $\operatorname{Rep}_{\mathbf{d}} F$ be the set of all algebra maps $F \rightarrow \mathbf{E}_{\mathbf{d}}:=\operatorname{Hom}_{\mathbb{C}}\left(\mathbb{C}^{\mathbf{d}}, \mathbb{C}^{\mathbf{d}}\right)$ which restrict to the natural map $R \rightarrow \mathbf{E}_{\mathbf{d}}$. The set $\operatorname{Rep}_{\mathbf{d}} F$ has the natural structure of a (not necessarily reduced) affine scheme of finite type, called the representation scheme 
of $\mathbf{d}$-dimensional representations of the algebra $F$. Let $\mathbb{C}\left[\operatorname{Rep}_{\mathbf{d}} F\right]$ denote the coordinate ring of this scheme.

2.2. Main result. Let $T_{R} V=R \oplus V \bigoplus V \otimes_{R} V \oplus \ldots$, denote the tensor algebra of an $R$-bimodule $V$, and write $T_{R}^{+} V=V \bigoplus V \otimes_{R} V \bigoplus \ldots$, for the augmentation ideal of $T_{R} V$.

Definition 2.2.1. A pair of finite dimensional $\mathbb{N}$-graded $R$-bimodules $V$ and $L$, together with a graded $R$-bimodule imbedding $j: L \hookrightarrow T_{R}^{+} V$, will be referred to as $(V, L)$-datum.

Fix a $(V, L)$-datum, where $V=\bigoplus_{r \geq 1} V[r]$, and $L=\bigoplus_{r \geq 1} L[r]$. We define

$$
L^{\circ}:=j^{-1}\left(\left[T_{R} V, T_{R} V\right]\right) \subset L .
$$

Thus, $L^{\circ}=\bigoplus_{r \geq 1} L^{\circ}[r]$ is a graded subspace in $L$. We put $m_{r}=\operatorname{dim} L^{\circ}[r]$, and let

$$
\lambda\left(L^{\circ}\right):=\prod_{r>0}\left(1-t^{r}\right)^{m_{r}} \in \mathbb{Z}[t] .
$$

We also introduce the following generating $\zeta$-function

$$
\zeta(V, L):=\prod_{s=1}^{\infty} \frac{1}{\operatorname{det}\left(\mathbf{1}-h\left(V ; t^{s}\right)+h\left(L ; t^{s}\right)\right)} \in \mathbb{Z}[[t]] .
$$

The gradings on $V, L$, and $L^{\circ}$, give rise to total gradings on various objects like exterior algebra $\Lambda L^{\circ}$, tensor algebra $T_{R} V$, etc. These total gradings will be called weight gradings. For the corresponding bigraded algebras, we write e.g., $\Lambda L^{\circ}=\bigoplus_{p, r \geq 0}\left(\Lambda^{p} L^{\circ}\right)[r]$. With this notation, for the polynomial in (2.2.3), one has

$$
\lambda\left(L^{\circ}\right)=\sum_{p, r}(-1)^{p} \cdot t^{r} \cdot \operatorname{dim}\left(\left(\Lambda^{p} L^{\circ}\right)[r]\right) .
$$

Associated with a $(V, L)$-datum, is the two-sided ideal $J:=(L) \subset T_{R} V$ generated by $j(L)$, and the corresponding quotient algebra $A:=T_{R} V / J=T_{R} V /(L)$. The weight grading on $T_{R} V$ makes $J$ a graded ideal and $A$ a graded algebra, $A=\bigoplus_{r \geq 0} A[r]$. For each $r \geq 0$, the homogeneous component $A[r]$ is a finite dimensional $R$-bimodule; we have $A[0]=R$.

Given an integer $N$ and a dimension vector $\mathbf{d}$, we write $\mathbf{d} \succ N$ if $d_{i}>N$ for all $i \in I$.

Definition 2.2.5. $A(V, L)$-datum is called representation complete intersection (RCI) if for any integer $N$ there exists a dimension vector $\mathbf{d} \succ N$ such that, cf. (2.1.2):

$$
\operatorname{dim} \operatorname{Rep}_{\mathbf{d}} A=\operatorname{dim} \mathbb{V}_{\mathbf{d}}-\operatorname{dim} \mathbb{L}_{\mathbf{d}}+\operatorname{dim} L^{\circ}
$$


The geometric meaning of equation (2.2.6) will be explained in $\S 2.3$ below.

Abusing terminology, we often say that the algebra $A=T_{R} V /(L)$ arising from the $(V, L)$-datum is an RCI algebra.

One of our main results reads

Theorem 2.2.7. Let $(V, L)$ be an $R C I$ datum and $A=T_{R} V /(L)$. Then, we have $H H_{k}(A)=0$ for all $k>2$ (i.e., $A$ is a NCCI algebra, cf. §3.1), and $H_{2}(A) \cong L^{\circ}$.

Furthermore, there is a natural exact sequence

$$
0 \longrightarrow R \longrightarrow H H_{0}(A) \longrightarrow H H_{1}(A) \longrightarrow H_{2}(A) \longrightarrow 0 .
$$

The Hilbert series of $A$, resp. of $H_{0}(A)=A /[A, A]$, is determined by the formula

(i) $h(A)=(\mathbf{1}-h(V)+h(L))^{-1}, \quad$ resp., $\quad$ (ii) $h(\mathcal{O}(A))=\zeta(V, L) / \lambda\left(L^{\circ}\right)$.

Remark 2.2.10. One may call $\mathbf{p}(V, L ; t):=\mathbf{1}-h(V ; t)+h(L ; t)$, a $\mathbb{Z}[t]$-valued $I \times I$-matrix, the Cartan polynomial of the $(V, L)$-datum. Thus, formulas (2.2.9) read

$$
h(A)=\mathbf{p}(V, L ; t)^{-1}, \quad \text { resp., } \quad h(\mathcal{O}(A))=\frac{1}{\lambda\left(L^{\circ}\right)} \cdot \prod_{s=1}^{\infty} \frac{1}{\operatorname{det} \mathbf{p}\left(V, L ; t^{s}\right)} .
$$

Remark 2.2.11. It follows from the isomorphism $L^{\circ} \cong H H_{2}(A)$ and from $(2.2 .8)$ that one has $h\left(H H_{1}(A)\right)=h\left(A_{+} /[A, A]\right)+h\left(L^{\circ}\right)$. Therefore, the Theorem implies also that the Hilbert series of $H H_{1}(A)$ may be found from the formula

$$
h\left(\operatorname{Sym} H H_{1}(A)\right)=\zeta(V, L) / \lambda\left(L^{\circ}\right)^{2} .
$$

The proof of Theorem 2.2.7 will be completed in $\S 4$. We provide, in effect, two different proofs of the Theorem. The first proof is based on a matrix integral calculation, to be carried out in $\S \S 2.3-2.4$ below. The second, purely algebraic proof, is based on the formula for $h(\mathcal{O}(A))$ in the special case of a free $R$-algebra $A$. Such a formula is known, it can be obtained by a combinatorial argument that involves counting cyclic paths on a graph, cf. [St]. The general case of Theorem 2.2.7 for an arbitrary RCI algebra $A$ can then be deduced from the special case of free $R$-algebras by applying the formula proved in the special case to the noncommutative Koszul complex $K$. $A$, which is free as an $R$-algebra, cf. §3.2.

2.3. Hilbert series of representation schemes. Fix a dimension vector $\mathbf{d}$ and a $(V, L)$-datum. Clearly, one has a natural isomorphism $\operatorname{Rep}_{\mathbf{d}}\left(T_{R} V\right) \cong$ $\operatorname{Hom}_{R \text {-bimod }}\left(V, \mathbf{E}_{\mathbf{d}}\right)=\mathbb{V}_{\mathbf{d}}$, cf. (2.1.2). 
Now, let $A=T_{R} V /(L)$. The algebra projection $T_{R} V \rightarrow A$ induces a natural closed imbedding of representation schemes $\operatorname{Rep}_{\mathbf{d}} A \hookrightarrow \operatorname{Rep}_{\mathbf{d}}\left(T_{R} V\right)$. Dually, the $R$-bimodule imbedding $j: L \hookrightarrow T_{R} V$ induces a natural restriction morphism

$$
j_{\mathbf{d}}^{*}: \mathbb{V}_{\mathbf{d}}=\operatorname{Hom}_{R \text {-bimod }}\left(V, \mathbf{E}_{\mathbf{d}}\right) \cong \operatorname{Rep}_{\mathbf{d}}\left(T_{R} V\right) \longrightarrow \mathbb{L}_{\mathbf{d}} .
$$

It is clear that the scheme $\operatorname{Rep}_{\mathbf{d}} A$ is the scheme-theoretic zero fiber of the morphism $j_{\mathbf{d}}^{*}$.

In general, the map $j_{\mathbf{d}}^{*}$ is not surjective. Indeed, for any algebra homomorphism $\rho: T_{R} V \rightarrow \mathbf{E}_{\mathbf{d}}$ and any $x \in\left[T_{R} V, T_{R} V\right]$, we have $\operatorname{Tr} \rho(x)=0$. In particular, the composite map

$$
L^{\circ} \stackrel{j}{\hookrightarrow}\left[T_{R} V, T_{R} V\right] \hookrightarrow T_{R} V \stackrel{\rho}{\longrightarrow} \mathbf{E}_{\mathbf{d}} \stackrel{\operatorname{Tr}}{\longrightarrow} \mathbb{C}
$$

must vanish. Therefore, the image of the map $j_{\mathbf{d}}^{*}$ in (2.3.1) is contained in a proper subspace $\mathbb{L}_{\mathbf{d}}^{\sharp}:=\operatorname{Ker} \psi \subset \mathbb{L}_{\mathbf{d}}$, the kernel of the following composite map

$$
\mathbb{L}_{\mathbf{d}}^{\sharp}:=\operatorname{Ker}\left[\psi: \mathbb{L}_{\mathbf{d}} \stackrel{\operatorname{res}_{L \circ}^{L}}{\longrightarrow} \operatorname{Hom}\left(L^{\circ}, \mathbf{E}_{\mathbf{d}}\right) \stackrel{\left.\operatorname{Tr}\right|_{\mathbf{E}_{\mathbf{d}}}}{\longrightarrow} \operatorname{Hom}\left(L^{\circ}, \mathbb{C}\right)=\left(L^{\circ}\right)^{*}\right] .
$$

Write $p_{\mathbf{d}}: \operatorname{Rep}_{\mathbf{d}}\left(T_{R} V\right) \rightarrow \mathbb{L}_{\mathbf{d}}^{\sharp}$ for the resulting map and let $o$ denote the origin of the vector space $\mathbb{L}_{\mathbf{d}}^{\sharp}$. This way, we get the following commutative diagram

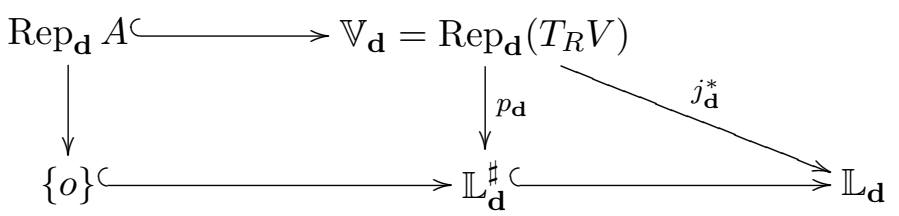

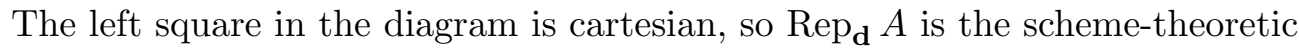
zero fiber of the morphism $p_{\mathbf{d}}$. The following result provides a geometric meaning for the notion of representation complete intersection datum.

Lemma 2.3.4. The dimension equality (2.2.6) holds for a dimension vector $\mathbf{d}$ if and only if the map $p_{\mathbf{d}}$, in diagram (3.3.4), is flat.

To prove the Lemma, we observe that the gradings on $V$ and $L$ make each of the spaces in diagram (2.3.3) a $\mathbb{C}^{\times}$-variety, such that all maps in the diagram become $\mathbb{C}^{\times}$-equivariant morphisms. Moreover, the $\mathbb{C}^{\times}$-action on $\mathbb{L}_{\mathbf{d}}^{\sharp}$ being a contraction, we deduce that the dimension of any fiber of the map $p_{\mathbf{d}}$ is less than or equal to

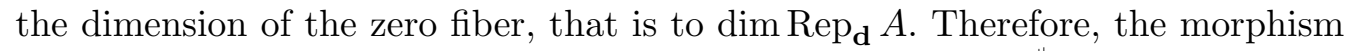
$p_{\mathbf{d}}$ is flat if and only if one has $\operatorname{dim}_{\operatorname{Rep}} A=\operatorname{dim} \mathbb{V}_{\mathbf{d}}-\operatorname{dim} \mathbb{L}_{\mathbf{d}}^{\sharp}$. 
Observe next that the map $\psi$ in (2.3.2) is surjective. Hence, one has an exact sequence

$$
0 \longrightarrow \mathbb{L}_{\mathbf{d}}^{\sharp} \longrightarrow \mathbb{L}_{\mathbf{d}} \stackrel{\psi}{\longrightarrow}\left(L^{\circ}\right)^{*} \longrightarrow 0 .
$$

We deduce: $\operatorname{dim} \mathbb{L}_{\mathbf{d}}^{\sharp}=\operatorname{dim} \mathbb{L}_{\mathbf{d}}-\operatorname{dim} L^{\circ}$, hence, $\operatorname{dim} \mathbb{V}_{\mathbf{d}}-\operatorname{dim} \mathbb{L}_{\mathbf{d}}^{\sharp}=\operatorname{dim} \mathbb{V}_{\mathbf{d}}-$ $\operatorname{dim} \mathbb{L}_{\mathbf{d}}+\operatorname{dim} L^{\circ}$, and the Lemma follows.

Given $i \in I$ and an element $g_{i} \in G L\left(\mathbb{C}^{d_{i}}\right)$, we write $g_{i}^{\vee}=\left(g_{i}^{\top}\right)^{-1}:\left(\mathbb{C}^{d_{i}}\right)^{*} \rightarrow$ $\left(\mathbb{C}^{d_{i}}\right)^{*}$, where $g_{i}^{\top}$ denotes the dual endomorphism of the dual vector space. For each pair of vertices $i, j \in I$, we consider endomorphisms of the vector space $\left(\mathbb{C}^{d_{i}}\right)^{*} \otimes \mathbb{C}^{d_{j}}=\operatorname{Hom}\left(\mathbb{C}^{d_{i}}, \mathbb{C}^{d_{j}}\right)=\mathbf{E}_{i j}$ of the form $g_{i}^{\vee} \otimes g_{j}$. Thus, given $g_{i} \in G L\left(\mathbb{C}^{d_{i}}\right)$ and $g_{j} \in G L\left(\mathbb{C}^{d_{j}}\right)$, for any integer $r \geq 0$, there is a well defined polynomial

$$
\operatorname{det}\left(\mathbf{1}_{\mathbf{E}_{i j}}-t^{r} \cdot g_{i}^{\vee} \otimes g_{j}\right) \in \mathbb{C}[t] .
$$

We put $G_{\mathbf{d}}=\prod_{i \in I} G L\left(\mathbb{C}^{d_{i}}\right)$. This is a reductive group that acts naturally on the vector space $\mathbb{C}^{\mathbf{d}}=\bigoplus_{i \in I} \mathbb{C}^{d_{i}}$, hence, also on the algebra $\mathbf{E}_{\mathbf{d}}$, by conjugation.

Next, for each $d$, we fix a maximal compact subgroup $U(d) \subset G L\left(\mathbb{C}^{d}\right)$. The group $U_{\mathbf{d}}:=\prod_{i \in I} U\left(d_{i}\right)$ is a maximal compact subgroup of $G_{\mathbf{d}}$. Let $d g$ be the Haar measure on $U_{\mathbf{d}}$ normalized so that the total volume of $U_{\mathbf{d}}$ equals 1.

We introduce the following matrix integral

$$
\mathbf{I}(V, L, \mathbf{d}):=\int_{U_{\mathbf{d}}} \prod_{r>0} \prod_{i, j \in I} \frac{d g}{\operatorname{det}\left(\mathbf{1}_{\mathbf{E}_{i j}}-t^{r} \cdot g_{i}^{\vee} \otimes g_{j}\right)^{\mathbf{c}^{r}(V, L)}} .
$$

Here, $\mathbf{c}_{i j}^{r}(V, L):=\operatorname{dim} V_{i j}[r]-\operatorname{dim} L_{i j}[r]$, and the above expression is viewed as a formal power series $\mathbf{I}(V, L, \mathbf{d})=\sum_{m>0} \mathbf{I}_{m}(V, L, \mathbf{d}) \cdot t^{m} \in \mathbb{C}[[t]]$ given by the power series expansion of the RHS of (2.3.6).

Conjugation-action of the group $G_{\mathbf{d}}$ on $\mathbf{E}_{\mathbf{d}}$ induces linear $G_{\mathbf{d}}$-actions on the vector spaces $\mathbb{V}_{\mathbf{d}}, \mathbb{L}_{\mathbf{d}}$, etc. Furthermore, all the maps in diagram (3.3.4) are $G_{\mathbf{d}}$-equivariant morphisms. In particular, the scheme $\operatorname{Rep}_{\mathbf{d}} A$ is a $G_{\mathbf{d}}$-stable subscheme of $\mathbb{V}_{\mathbf{d}}$, so the group $G_{\mathbf{d}}$ acts on the coordinate ring $\mathbb{C}\left[\operatorname{Rep}_{\mathbf{d}} A\right]$ by algebra automorphisms.

We also have the $\mathbb{C}^{\times}$-action on the scheme $\operatorname{Rep}_{\mathbf{d}} A \subset \mathbb{V}_{\mathbf{d}}$ that commutes with the $G_{\mathbf{d}}$-action. The induced $\mathbb{C}^{\times}$-action on the subalgebra of $G_{\mathbf{d}}$-invariants gives rise to a grading

$$
\mathbb{C}\left[\operatorname{Rep}_{\mathbf{d}} A\right]^{G_{\mathbf{d}}}=\bigoplus_{r \geq 0} \mathbb{C}\left[\operatorname{Rep}_{\mathbf{d}} A\right]^{G_{\mathbf{d}}}[r],
$$

to be referred to as weight grading.

Proposition 2.3.8. Let $A=T_{R} V$ be the graded algebra associated to a $(V, L)$ datum. Assume that the map $p_{\mathbf{d}}$ in (2.3.3) is flat, so the scheme $\operatorname{Rep}_{\mathbf{d}} A$ is 
a complete intersection in $\mathbb{V}_{\mathbf{d}}$. Then, the Hilbert series of the graded algebra $\mathbb{C}\left[\operatorname{Rep}_{\mathbf{d}} A\right]^{G_{\mathbf{d}}}$ is given by

$$
h\left(\mathbb{C}\left[\operatorname{Rep}_{\mathbf{d}} A\right]^{G_{\mathbf{d}}}\right)=\mathbf{I}(V, L, \mathbf{d}) / \lambda\left(L^{\circ}\right) .
$$

Proof. Associated with the zero fiber of the map $p_{\mathbf{d}}$, see (2.3.3), is the standard Koszul complex with differential $d_{K}$ of degree -1 :

$$
\begin{gathered}
\text { K. }\left(\operatorname{Rep}_{\mathbf{d}} A\right)=\mathbb{C}\left[\mathbb{V}_{\mathbf{d}}\right] \otimes \Lambda^{\bullet}\left(\mathbb{L}_{\mathbf{d}}^{\sharp}\right)^{*}, \quad \text { such that } \\
\mathbb{C}\left[\operatorname{Rep}_{\mathbf{d}} A\right]=\operatorname{Coker}\left[d_{K}: \mathbf{K}_{1}\left(\operatorname{Rep}_{\mathbf{d}} A\right) \rightarrow \mathbf{K}_{0}\left(\operatorname{Rep}_{\mathbf{d}} A\right)\right] .
\end{gathered}
$$

For each $p \geq 0$, the $\mathbb{C}^{\times}$-action on $\mathbb{V}_{\mathbf{d}}$ and on $\mathbb{L}_{\mathbf{d}}^{\sharp}$ gives an additional weight grading on the vector space $\mathbf{K}_{p}\left(\operatorname{Rep}_{\mathbf{d}} A\right)$. The Koszul differential respects the weight grading. Writing $[r]$ to denote weight $r$ homogeneous component, we get a bigraded direct sum decomposition

$$
\text { K. }\left(\operatorname{Rep}_{\mathbf{d}} A\right)=\bigoplus_{p, r \geq 0} \mathbf{K}_{p}\left(\operatorname{Rep}_{\mathbf{d}} A\right)[r] .
$$

Observe next that all the maps in (2.3.5) are $G_{\mathbf{d}^{-}}$equivariant, provided one treats $\left(L^{\circ}\right)^{*}$ as a vector space with the trivial $G_{\mathbf{d}^{-}}$-action. Therefore, dualizing (2.3.5) yields a $G_{\mathbf{d}^{-}}$equivariant short exact sequence

$$
0 \longrightarrow L^{\circ} \stackrel{\psi^{\top}}{\longrightarrow} \mathbb{L}_{\mathbf{d}}^{*} \longrightarrow\left(\mathbb{L}_{\mathbf{d}}^{\sharp}\right)^{*} \longrightarrow 0 .
$$

Thus, one can rewrite the Koszul complex in the form

$$
\text { K. }\left(\operatorname{Rep}_{\mathbf{d}} A\right)=\mathbb{C}\left[\mathbb{V}_{\mathbf{d}}\right] \otimes \Lambda^{\bullet}\left(\mathbb{L}_{\mathbf{d}}^{*} / L^{\circ}\right) .
$$

Assume now that the map $p_{\mathbf{d}}$ in (3.3.4) is flat so that the scheme $\operatorname{Rep}_{\mathbf{d}} A$ is a complete intersection. Then, the Koszul complex provides a graded, $G_{\mathbf{d}^{-}}$ equivariant DG algebra resolution of the coordinate ring $\mathbb{C}\left[\operatorname{Rep}_{\mathbf{d}} A\right]$. It follows, since the group $G_{\mathbf{d}}$ is reductive, that the $G_{\mathbf{d}}$-fixed part of the Koszul complex provides a graded DG algebra resolution of $\mathbb{C}\left[\operatorname{Rep}_{\mathbf{d}} A\right]^{G_{\mathbf{d}}}$, the subalgebra of $G_{\mathbf{d}^{-}}$ invariants in $\mathbb{C}\left[\operatorname{Rep}_{\mathbf{d}} A\right]$. Thus, by the Euler-Poincaré principle, the Hilbert series of $\mathbb{C}\left[\operatorname{Rep}_{\mathbf{d}} A\right]^{G_{\mathbf{d}}}$ may be expressed in terms of Hilbert series of the graded spaces $\mathbf{K}_{p}\left(\operatorname{Rep}_{\mathbf{d}} A\right)^{G_{\mathbf{d}}}=\bigoplus_{r \geq 0} \mathbf{K}_{p}\left(\operatorname{Rep}_{\mathbf{d}} A\right)^{G_{\mathbf{d}}}[r]$ as follows

$$
\begin{aligned}
h\left(\mathbb{C}\left[\operatorname{Rep}_{\mathbf{d}} A\right]^{G_{\mathbf{d}}}\right) & =\sum_{p \geq 0}(-1)^{p} \cdot h\left(\mathbf{K}_{p}\left(\operatorname{Rep}_{\mathbf{d}} A\right)^{G_{\mathbf{d}}}\right) \\
& =\sum_{p \geq 0}(-1)^{p} \cdot h\left(\left(\mathbb{C}\left[\mathbb{V}_{\mathbf{d}}\right] \otimes \Lambda^{p}\left(\mathbb{L}_{\mathbf{d}}^{*} / L^{\circ}\right)\right)^{G_{\mathbf{d}}}\right) .
\end{aligned}
$$

To compute the last expression, recall that for any two linear maps, $u: M \rightarrow M$ and $v: N \rightarrow N$, of finite dimensional vector spaces, one has

$$
\sum_{p, m \geq 0}(-1)^{p} \cdot t^{m} \cdot \operatorname{Tr}\left(\left.v \otimes u\right|_{\operatorname{Sym}^{m} N \otimes \Lambda^{p} M}\right)=\frac{\operatorname{det}(\mathbf{1}-t \cdot u)}{\operatorname{det}(\mathbf{1}-t \cdot v)} .
$$


Now, for any $p \geq 0$, we have a direct sum decomposition with respect to the weight grading

$$
\mathbb{C}\left[\mathbb{V}_{\mathbf{d}}\right] \otimes \Lambda^{p}\left(\mathbb{L}_{\mathbf{d}}^{*} / L^{\circ}\right)=\bigoplus_{r \geq 0}\left(\mathbb{C}\left[\mathbb{V}_{\mathbf{d}}\right] \otimes \Lambda^{p}\left(\mathbb{L}_{\mathbf{d}}^{*} / L^{\circ}\right)\right)[r]
$$

Thus, using the above formula and the notation $\mathbf{c}_{i j}^{r}=\operatorname{dim} V_{i j}[r]-\operatorname{dim} L_{i j}[r]$, for any $g \in G_{\mathbf{d}}$, we find

$$
\begin{aligned}
& (2 . \operatorname{S.1} 1 \xi+1)^{p} \cdot t^{r} \cdot \operatorname{Tr}\left(\left.g\right|_{\left(\mathbb{C}\left[\mathbb{V}_{\mathbf{d}}\right] \otimes \Lambda^{p}\left(\mathbb{L}_{\mathbf{d}}^{*} / L^{\circ}\right)\right)[r]}\right) \\
= & \prod_{r>0}\left(\frac{1}{\left(1-t^{r}\right)^{m_{r}}} \cdot \prod_{i, j \in I} \frac{\operatorname{det}\left(\mathbf{1}-\left.t^{r} g\right|_{\mathbf{E}_{i j}}\right)^{\operatorname{dim} L_{i j}[r]}}{\operatorname{det}\left(\mathbf{1}-\left.t^{r} g\right|_{\mathbf{E}_{i j}}\right)^{\operatorname{dim} V_{i j}[r]}}\right)=\frac{1}{\lambda\left(L^{\circ} \prod_{i, j \in I}\right.} \frac{1}{\operatorname{det}\left(\mathbf{1}-\left.t^{r} g\right|_{\mathbf{E}_{i j}}\right)^{\mathbf{c}_{i j}^{r}}} .
\end{aligned}
$$

Recall that, for any finite dimensional $G_{\mathbf{d}}$-module $M$, the dimension of the $G_{\mathbf{d}}$-fixed point subspace is given by the integral $\operatorname{dim} M^{G_{\mathbf{d}}}=\int_{U_{\mathbf{d}}} \operatorname{Tr}\left(\left.g\right|_{M}\right) d g$. We apply this to each direct summand of the graded $G_{\mathbf{d}}$-module in the right-hand side of (2.3.14). We conclude that the Hilbert series of the subcomplex of $G_{\mathbf{d}^{-}}$ invariants from the last line in (2.3.13) is obtained by averaging the expression in (2.3.15) over $U_{\mathbf{d}}$, which gives the desired formula.

2.4. Asymptotics of the matrix integral. We will use the following result about random unitary matrices (see e.g. [DS]).

Proposition 2.4.1. Let $m_{s}, n_{s}, s \geq 1$, be nonnegative integers, which are almost all zero. Then for large enough $N$,

$$
\int_{U(N)} \prod_{s \geq 1}\left(\operatorname{Tr}\left(u^{s}\right)\right)^{m_{s}}\left(\overline{\operatorname{Tr}\left(u^{s}\right)}\right)^{n_{s}} d u=\prod_{s \geq 1} s^{m_{s}} \cdot m_{s} ! \cdot \delta_{m_{s}, n_{s}}
$$

The statement of the proposition means that the functions $z_{s}(u)=\operatorname{Tr}\left(u^{s}\right) / \sqrt{s}$ behave, asymptotically as $N \rightarrow \infty$, as independent complex-valued random variables distributed with Gaussian density $p(z)=\pi^{-1} e^{-z \bar{z}}$. It follows, in particular, that one has

Corollary 2.4.2. For any finite collection of polynomial functions $f_{s} \in \mathbb{C}\left[x_{1}, x_{2}\right]$, $s=1,2, \ldots, m$, there exists $N \gg 0$ such that for all $d \geq N$ one has

$$
\int_{U(d)}\left(\prod_{s \geq 1}^{m} f_{s}\left(z_{s}(u), \overline{z_{s}(u)}\right)\right) d u=\prod_{s \geq 1}^{m}\left(\int_{U(d)} f_{s}\left(z_{s}(u), \overline{z_{s}(u)}\right) d u\right) .
$$


Moreover, we have

$$
\int_{U(d)} f_{s}\left(z_{s}(u), \overline{z_{s}(u)}\right) d u=\frac{1}{\pi} \int_{\mathbb{C}} f_{s}(z, \bar{z}) e^{-|z|^{2}} d z d \bar{z}, \quad \forall s=1,2, \ldots
$$

The statement of Corollary 2.4.2 can be generalized further to the case of integrals over $U_{\mathbf{d}}=\prod_{i \in I} U\left(d_{i}\right)$, a product of unitary groups. With such a generalization at hand, we are now ready to prove

Corollary 2.4.5. For each integer $m$, the sequence $\mathbf{I}_{m}(V, L, \mathbf{d})$, of $m$-th coefficients in the expansion (2.3.6), stabilizes as $\mathbf{d} \rightarrow \infty$. Write $\lim _{\mathbf{d} \rightarrow \infty} \mathbf{I}_{m}(V, L, \mathbf{d})$ for this stabilized coefficient and define a formal power series

$$
\lim _{\mathbf{d} \rightarrow \infty} \mathbf{I}(V, L, \mathbf{d}):=\sum_{m \geq 0} t^{m} \cdot \lim _{\mathbf{d} \rightarrow \infty} \mathbf{I}_{m}(V, L, \mathbf{d}) .
$$

Then, we have $\lim _{\mathbf{d} \rightarrow \infty} \mathbf{I}(V, L, \mathbf{d})=\zeta(V, L)$.

Proof. We have the standard identity

$\operatorname{det}(\operatorname{Id}-u)=\exp (\operatorname{Tr} \log (\operatorname{Id}-u))=\exp \left(\sum_{s \geq 1} \frac{1}{s} \operatorname{Tr}\left(u^{s}\right)\right)=\prod_{s \geq 1} \exp \left(\frac{1}{s} \operatorname{Tr}\left(u^{s}\right)\right)$.

We apply this identity to the function under the integral sign in formula (2.3.6) and get

$$
\prod_{r>0} \prod_{i, j \in I} \frac{1}{\operatorname{det}\left(\mathbf{1}_{\mathbf{E}_{i j}}-t^{r} \cdot g_{i}^{\vee} \otimes g_{j}\right)^{\mathbf{c}_{i j}^{r}}}=\prod_{s \geq 1} \exp \left(\sum_{i, j \in I, r \in \mathbb{N}} \mathbf{c}_{i j}^{r} \operatorname{Tr}\left(g^{s} \mid \mathbf{E}_{i j}\right) \cdot \frac{t^{r s}}{s}\right)
$$

Given $g=\left\{g_{i}\right\}_{i \in I} \in U_{\mathbf{d}}$, let $z_{i s}(g)$ denote the function $g \mapsto z_{i s}(g)=\operatorname{Tr}\left(g_{i}^{s} \mid \mathbf{E}_{i i}\right) / \sqrt{s}$. According to formla (2.3.6), using (2.4.6), we find

$$
\mathbf{I}(V, L, \mathbf{d} ; t)=\int_{U_{\mathbf{d}}}\left(\prod_{s \geq 1} \exp \left(\sum_{i, j \in I, r \in \mathbb{N}} \mathbf{c}_{i j}^{r} \cdot t^{r s} \cdot z_{i s}(g) \overline{z_{j s}(g)}\right)\right) .
$$

Now, using an analogue of (2.4.3) for the group $U_{\mathbf{d}}$, in the limit $d_{i} \rightarrow \infty, i \in I$, from (2.4.7) we obtain

$$
\lim _{\mathbf{d} \rightarrow \infty} \mathbf{I}(V, L, \mathbf{d} ; t)=\prod_{s \geq 1}\left(\int_{U_{\mathbf{d}}} \exp \left(\sum_{i, j \in I, r \in \mathbb{N}} \mathbf{c}_{i j}^{r} \cdot t^{r s} \cdot z_{i s}(g) \overline{z_{j s}(g)}\right)\right)=\prod_{s \geq 1} f_{s}(t),
$$


where the functions $f_{s}$ are given by an analogue of formula (2.4.4), that is,

$$
\begin{aligned}
f_{s}(t) & =\frac{1}{\pi^{|I|}} \int_{\mathbb{C}^{I}} \exp \left(\sum_{i, j \in I, r \in \mathbb{N}} \mathbf{c}_{i j}^{r} \cdot t^{r s} \cdot z_{i} \overline{z_{j}}\right) \cdot e^{-\sum_{k \in I}\left|z_{k}\right|^{2}} \\
& =\frac{1}{\pi^{|I|}} \int_{\mathbb{C}^{I}} \exp \left(-\sum_{i, j \in I, r \in \mathbb{N}}\left(\delta_{i j}-\mathbf{c}_{i j}^{r} \cdot t^{r s}\right) \cdot z_{i} \overline{z_{j}}\right)=\frac{1}{\operatorname{det}\left(\mathbf{1}-\sum_{r>0} \mathbf{c}_{i j}^{r} \cdot t^{r s}\right)} .
\end{aligned}
$$

Finally, the last expression on the right equals $1 / \operatorname{det}\left(\mathbf{1}-h\left(V ; t^{s}\right)+h\left(L ; t^{s}\right)\right)$, by definition of the coefficients $\mathbf{c}_{i j}^{r}:=\operatorname{dim} V_{i j}[r]-\operatorname{dim} L_{i j}[r]$.

Definition 2.4.8. $A(V, L)$-datum (or the algebra $A=T_{R} V /(L)$ ) is called an asymptotic RCI if, for any pair of positive integers $r, N$, there exists a dimension vector $\mathbf{d} \succ N$ such that one has:

$$
H_{p}\left(\bigoplus_{s \leq r} \mathbf{K} \cdot\left(\operatorname{Rep}_{\mathbf{d}} A\right)[s], d_{K}\right)=0, \quad \forall p>0,
$$

i.e., such that all nonzero homology groups of the weight degree $\leq r$ part of the Koszul complex (2.3.10) vanish.

Clearly, any RCI algebra is an asymptotic RCI; we will see in $\S 5$ below that the class of RCI algebras is rather restrictive while the class of asymptotic RCI is much less restrictive.

Let $A$ be an asymptotic RCI, and fix a positive integer $r$. Combining together Proposition 2.3.8 and Corollary 2.4.5, we deduce that there exists a sequence of dimension vectors $\mathbf{d}_{1}, \mathbf{d}_{2}, \ldots$, such that $\mathbf{d}_{m} \rightarrow \infty$ and such that the integer valued function $m \mapsto \operatorname{dim}\left(\mathbb{C}\left[\operatorname{Rep}_{\mathbf{d}_{m}} A\right]^{G_{\mathbf{d}}}[r]\right)$ stabilizes as $m \rightarrow \infty$ (we will see later, in Proposition 4.2.2, that this stabilization is a general phenomenon that holds for all large enough dimensions $\mathbf{d}$ and, moreover, has nothing to do with the RCI property). Write $\lim _{\mathbf{d} \rightarrow \infty} \operatorname{dim}\left(\mathbb{C}\left[\operatorname{Rep}_{\mathbf{d}} A\right]^{G_{\mathbf{d}}}[r]\right)$ for this stable value and define a power series

$$
\lim _{\mathbf{d} \rightarrow \infty} h\left(\mathbb{C}\left[\operatorname{Rep}_{\mathbf{d}} A\right]^{G_{\mathbf{d}}}\right):=\sum_{r \geq 0} t^{r} \cdot \lim _{\mathbf{d} \rightarrow \infty} \operatorname{dim}\left(\mathbb{C}\left[\operatorname{Rep}_{\mathbf{d}} A\right]^{G_{\mathbf{d}}}[r]\right) .
$$

Combining together Propositions 2.3.8 and 2.4.1, we obtain the following result

Proposition 2.4.9. For any asymptotic RCI algebra $A=T_{R} V /(L)$ we have, cf. $(2.2 .9)$ (ii)

$$
\lim _{\mathbf{d} \rightarrow \infty} h\left(\mathbb{C}\left[\operatorname{Rep}_{\mathbf{d}} A\right]^{G_{\mathbf{d}}}\right)=\frac{1}{\lambda\left(L^{\circ}\right)} \cdot \lim _{\mathbf{d} \rightarrow \infty} \mathbf{I}(V, L, \mathbf{d})=\zeta(V, L) / \lambda\left(L^{\circ}\right) .
$$

\section{Noncommutative COMPlete intersections}


3.1. NCCI algebras. Below, we consider nonnegatively graded algebras $A=$ $\bigoplus_{r \geq 0} A[r]$ such that $A[0]=R$, to be referred as $R$-algebras. We write $A_{+}=$ $\bigoplus_{r>0} A[r]$, so $A / A_{+}=R$.

For any $R$-bimodule $V$ and any two-sided ideal $J \subset T_{R}^{+} V$, the quotient $J / J^{2}$ has an obvious structure of $T_{R} V / J$-bimodule.

Let $V$ be an $\mathbb{N}$-graded finite dimensional $R$-bimodule and $J \subset T_{R}^{+} V$ a two-sided graded ideal. A graded algebra of the form $A=T_{R} V / J$ is called a noncommutative complete intersection (NCCI) algebra provided the equivalent conditions (i)-(iii) of the theorem below hold for $A$.

Theorem 3.1.1. Let $V$ be a finite dimensional $\mathbb{N}$-graded $R$-bimodule and $J \subset$ $T_{R} V$ a two-sided finitely generated graded ideal. The following properties of the graded algebra $A=T_{R} V / J$ are equivalent:

(i) $\mathrm{J} / \mathrm{J}^{2}$ is projective as a graded A-bimodule;

(ii) The algebra $A$ has Hochschild dimension $\leq 2$, i.e., one has $\operatorname{Ext}_{A \text {-bimod }}^{i}(A, M)=0$, for any $A$-bimodule $M$ and any $i \geq 3$;

(iii) The algebra $A$ has global dimension $\leq 2$, i.e., one has $\operatorname{Ext}_{A \text {-mod }}^{i}\left(A / A_{+}, M\right)=$ 0 , for any left $A$-module $M$ and any $i \geq 3$.

This result is known; its proof will be recalled in $\S 3.4$ below.

3.2. Anick's resolution and the noncommutative Koszul complex. Fix a $(V, L)$-datum and let $A=T_{R} V /(L)$.

Following [AH], [An], [GS], one introduces a noncommutative Koszul complex, $K . A$, (referred to as the Shafarevich complex in [Go], [Pi]), as follows. Set $K . A=$ $T_{R}(V \oplus L)$, and view this tensor algebra as a graded algebra with respect to homological grading, cf. $§ 3.5$, such that the vector space $V \subset T_{R}^{1}(V \oplus L)$ is placed in homological degree 0 and the vector space $L \subset T_{R}^{1}(V \oplus L)$ is placed in homological degree 1 . Thus, we have $K_{0} A=T_{R} V$. Define the map

$$
V \oplus L \longrightarrow T_{R}(V \oplus L), \quad v \oplus \ell \mapsto j(\ell) \in T_{R} V \subset T_{R}(V \oplus L)
$$

Let $d_{K}: K . A \rightarrow K_{{ }_{-1}} A$ be the unique super-derivation of the graded algebra $K$. $A$ that is given on the generators from $V \oplus L \subset K$. $A$ by formula (3.2.1). It is clear that we have $d_{K}^{2}=0$. Thus, $\left(K \cdot A, d_{K}\right)$ is a DG algebra. Write $H \cdot\left(K \cdot A, d_{K}\right)$ for the corresponding homology. Clearly we have $H_{0}\left(K . A, d_{K}\right)=A$. Therefore, the total homology space $H_{\bullet}\left(K . A, d_{K}\right)$ acquires the natural structure of a graded $A$-algebra. 
Next, we recall the definition of Anick's resolution [An2]. This is a complex of graded $A$-bimodules of the form

$$
C . A: A \otimes_{R} L \otimes_{R} A \stackrel{\partial}{\longrightarrow} A \otimes_{R} V \otimes_{R} A \stackrel{f}{\longrightarrow} A \otimes_{R} A \stackrel{\mathbf{m}_{A}}{\longrightarrow} A \rightarrow 0 .
$$

Here, $\mathbf{m}_{A}$ is the multiplication map, and the maps $\partial$ and $f$ are given by the formulas

$$
\partial\left(a_{1} \otimes \ell \otimes a_{2}\right)=a_{1} \cdot D(\ell) \cdot a_{2}, \quad \text { resp. } \quad f\left(a_{1} \otimes v \otimes a_{2}\right)=a_{1} v \otimes a_{2}-a_{1} \otimes v a_{2} .
$$

In the first formula above, we have used the map

$$
D: T_{R}^{+} V \rightarrow A \otimes_{R} V \otimes_{R} A, \quad v_{1} \otimes \ldots \otimes v_{n} \mapsto \sum_{p=1}^{n}\left(\overline{v_{1} \otimes \ldots \otimes v_{p-1}}\right) \otimes v_{p} \otimes\left(\overline{v_{p+1} \otimes \ldots \otimes v_{n}}\right),
$$

where bar stands for the image in $A$ of an element of $T_{R} V$.

Definition 3.2.3. $A(V, L)$-datum (or a graded $R$-bimodule $L \subset T_{R}^{+} V$ ) is said to be minimal provided one has

$$
\left(T_{R} V \cdot L \cdot T_{R}^{+} V+T_{R}^{+} V \cdot L \cdot T_{R} V\right) \cap L=0 .
$$

It is clear that, for any graded $R$-bimodule $L \subset T_{R}^{+} V$, one can always find a minimal sub-bimodule $\widetilde{L} \subset L$ such that $(L)=(\widetilde{L})$. Although the choice of such a minimal sub-bimodule $\widetilde{L}$ is not unique, any two minimal sub-bimodules $\widetilde{L}$ have the same Hilbert series.

For any $(V, L)$-datum, the composite $L \hookrightarrow J \rightarrow J / J^{2}$ extends, by $A$-linearity, to an $A$-bimodule map $\pi: A \otimes_{R} L \otimes_{R} A \rightarrow J / J^{2}$.

The proof of the following known (see e.g. [An]) result will be given in $§ 3.4$.

Theorem 3.2.4. The complex $C$. A is exact in all the terms except possibly the first one. Further, the following conditions are equivalent

(1) The bimodule $L$ is minimal and $A$ is an NCCI.

(2) The Hilbert series of $A$ equals $h(A)=(\mathbf{1}-h(V)+h(L))^{-1}$;

(3) The above defined natural map $\pi: A \otimes_{R} L \otimes_{R} A \rightarrow J / J^{2}$ is an isomorphism;

(4) The complex C.A is an A-bimodule resolution of $A$, i.e. the first map $\partial$ in (3.2.2) is injective;

(5) The Koszul complex K.A is a DG algebra resolution of $A$, i.e., we have $H_{k}(K . A, d)=0$, for any $k>0$.

We remark that if condition (5) holds then $K . A$ is, in effect, a minimal free associative DG algebra resolution of $A$ in the sense of minimal models, cf. [AH]. 
3.3. Noncommutative differential forms. Associated with an $R$-algebra $A$, is an $A$-bimodule $\Omega^{1} A$ of noncommutative relative 1 -forms on $A$ defined as the kernel of the multiplication map $\mathbf{m}_{A}: A \otimes_{R} A \rightarrow A$. Thus, one has a short exact sequence of $A$-bimodules

$$
0 \longrightarrow \Omega^{1} A \stackrel{\imath_{A}}{\longrightarrow} A \otimes_{R} A \stackrel{\mathbf{m}_{A}}{\longrightarrow} A \longrightarrow 0 .
$$

Next, let $F$ be an $R$-algebra, $J \subset F$ a two-sided ideal, and let $A:=F / J$. We put $\Omega^{1}(F \mid A):=A \otimes_{F} \Omega^{1} F \otimes_{F} A$. This is an $A$-bimodule equipped with an $A$-bimodule map $\tau: \Omega^{1}(F \mid A) \rightarrow \Omega^{1} A$, induced by the projection $F \rightarrow A$. There is a canonical short exact sequence of $A$-bimodules, cf. [CQ, Corollary 2.11],

$$
0 \longrightarrow J / J^{2} \stackrel{d}{\longrightarrow} \Omega^{1}(F \mid A) \stackrel{\tau}{\longrightarrow} \Omega^{1} A \longrightarrow 0 .
$$

Here, the map $J / J^{2} \rightarrow A \otimes_{F} \Omega^{1} F \otimes_{F} A=\Omega^{1}(F \mid A)$ is induced by restriction to $J$ of the de Rham differential $d: F \rightarrow \Omega^{1} F$. This exact sequence may be thought of as a noncommutative analogue of the conormal exact sequence of a subvariety.

We now fix a $(V, L)$-datum and set $F=T_{R} V, J=(L)$, and $A=T_{R} V /(L)$. Then we have natural isomorphisms

$$
\Omega^{1} F \cong F \otimes_{R} V \otimes_{R} F, \quad \text { and } \quad \Omega^{1}(F \mid A)=A \otimes_{R} V \otimes_{R} A .
$$

Splicing (3.3.2) and (3.3.1) together, we obtain the exact sequence in the bottom row of the following commutative diagram of $A$-bimodules

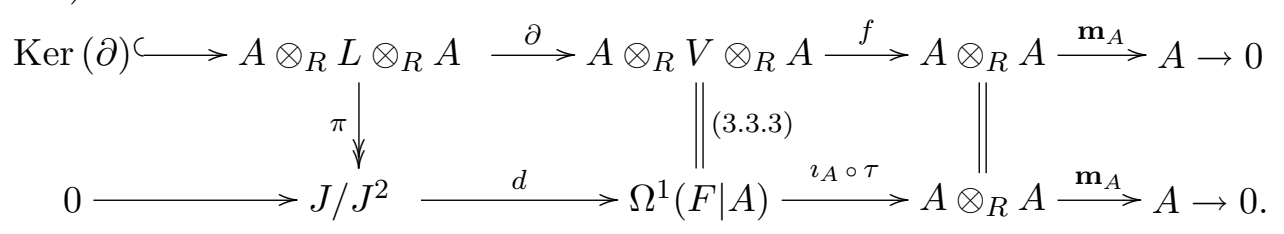

3.4. Proof of Theorem 3.1.1 and Theorem 3.2.4. The first claim of Theorem 3.2.4 amounts to the exactness of the top row of diagram (3.3.4). The latter follows from commutativity of the diagram since the bottom row of the diagram is exact. Also, from the commutative diagram we deduce $\operatorname{Ker} \partial \cong \operatorname{Ker} \pi$. This yields the equivalence $(3) \Leftrightarrow(4)$.

Next, we apply the Euler-Poincaré principle to the complex $C . A$ (in which the differential has degree 0 ), and find

$$
h(A) \cdot(\mathbf{1}-h(A) \cdot(\mathbf{1}-h(V)+h(L)))+h(\operatorname{Ker} \partial)=0 .
$$

Thus $h(\operatorname{Ker} \partial)=0$ if and only if $h(A)=(\mathbf{1}-h(V)+h(L))^{-1}$. This yields the equivalence $(2) \Longleftrightarrow(3)$. Also, it is clear that (2) implies property (i) of Theorem 3.1.1. 
Now, if $J / J^{2}$ is a projective $A$-bimodule, then the bottom row of diagram (3.3.4) provides a length 3 projective $A$-bimodule resolution of $A$. It follows that $A$ has Hochschild dimension $\leq 2$. Hence, in Theorem 3.1.1, we have (i) $\Rightarrow$ (ii). Similarly, we have (i) $\Rightarrow$ (iii). Conversely, from the exact sequence in the second row of diagram (3.3.4), for any $A$-bimodule $M$, we deduce $\operatorname{Ext}_{A \text {-bimod }}^{1}\left(J / J^{2}, M\right)=$ $\operatorname{Ext}_{A \text {-bimod }}^{3}(A, M)$. The latter Ext-group vanishes if $A$ has Hochschild dimension $\leq 2$. This yields the implication (ii) $\Rightarrow$ (i) in Theorem 3.1.1.

Any graded finitely generated projective $A$-bimodule has the form $A \otimes_{R} \widetilde{L} \otimes_{R}$ $A$, for some finitely generated graded $R$-bimodule $\widetilde{L}$. In particular, if $J / J^{2}$ is projective, we deduce

$$
\left(A / A_{+}\right) \otimes_{A}\left(J / J^{2}\right) \otimes_{A}\left(A / A_{+}\right)=\left(A / A_{+}\right) \otimes_{A}\left(A \otimes_{R} \widetilde{L} \otimes_{R} A\right) \otimes_{A}\left(A / A_{+}\right)=\widetilde{L} .
$$

Therefore, applying the functor $\left(A / A_{+}\right) \otimes_{A}(-) \otimes_{A}\left(A / A_{+}\right)$to the vertical map $\pi$ in diagram (3.3.4), we obtain a surjective $R$-bimodule map

$L=\left(A / A_{+}\right) \otimes_{A}\left(A \otimes_{R} L \otimes_{R} A\right) \otimes_{A}\left(A / A_{+}\right) \rightarrow\left(A / A_{+}\right) \otimes_{A}\left(J / J^{2}\right) \otimes_{A}\left(A / A_{+}\right)=\widetilde{L}$.

The resulting map $L \rightarrow \widetilde{L}$ may be identified with the composite map

$L \hookrightarrow J \rightarrow \frac{J}{F_{+} \cdot J+J \cdot F_{+}}=\frac{J}{J^{2}+F_{+} \cdot J+J \cdot F_{+}}=\left(A / A_{+}\right) \otimes_{A}\left(J / J^{2}\right) \otimes_{A}\left(A / A_{+}\right)$.

Observe that the bimodule $L$ is minimal if and only if the latter map is a bijection. Since $L$ is minimal by assumptions, we deduce that the map $\pi$ induces an isomorphism $L \simeq \widetilde{L}$. It follows that the vertical map $\pi$ in diagram (3.3.4) is itself an isomorphism, and we have proved the equivalence $(1) \Longleftrightarrow(3)$.

The equivalence $(1) \Longleftrightarrow(5)$, as well as the implication (i) $\Rightarrow$ (iii) of Theorem 3.1.1, are due to Anick, see Theorem 2.6 and Corollary 2.12(b) in [An]; cf. also Remark 5.3.5 in [Gi3] for an alternative approach.

The implication $(5) \Rightarrow(2)$ has been observed first by Golod-Shafarevich [GS].

3.5. A super-version. Given a $\mathbb{Z} / 2 \mathbb{Z}$-graded super-vector space $M=M_{\text {even }} \oplus$ $M_{\text {odd }}$, for the corresponding super-symmetric (supercommutative) algebra we introduce the notation

$$
\operatorname{Sym} M:=\left(\operatorname{Sym} M_{\text {even }}\right) \otimes\left(\Lambda M_{\text {odd }}\right) .
$$

If each of the spaces $M_{\text {even }}, M_{\text {odd }}$ is equipped with an additional grading, we say that $M$ is a graded super-vector space. In that case, we view Sym $M$ as a graded super-algebra equipped with the total grading.

Below, we will frequently encounter $b i$-graded vector spaces with two types of gradings of the form $M=\bigoplus_{p \in \mathbb{Z}, r \geq 0} M_{p}[r]$. The $p$-grading, written as $M=$ 
$\bigoplus_{p \in \mathbb{Z}} M_{p}$ where $M_{p}:=\bigoplus_{r} M_{p}[r]$, will be called homological grading. The $r$ grading, written as $M=\bigoplus_{r \geq 0} M[r]$ where $M[r]=\bigoplus_{p} M_{p}[r]$, will be called weight grading. In such a case, we put $M_{\text {even }}=\bigoplus_{p \text { even }} M_{p}$ and $M_{\text {odd }}=\bigoplus_{p \text { odd }} M_{p}$. The bigrading on $M$ induces corresponding homological and weight gradings $\operatorname{Sym} M=\bigoplus_{p \in \mathbb{Z}, r \geq 0}(\operatorname{Sym} M)_{p}[r]$. Thus, for any $k \geq 0$, we have

$m_{1} \& \ldots \& m_{k} \in(\operatorname{Sym} M)_{p_{1}+\ldots+p_{k}}\left[r_{1}+\ldots+r_{k}\right], \quad \forall m_{1} \in M_{p_{1}}\left[r_{1}\right], \ldots, m_{k} \in M_{p_{k}}\left[r_{k}\right]$.

Hilbert series will be always taken with respect to the weight grading (with parity being determined by the homological grading). Specifically, we define the Hilbert series of a bi-graded (super)-vector space $M=\bigoplus_{p \in \mathbb{Z}, r \geq 0} M_{p}[r]$ to be

$$
h(M ; t)=h\left(M_{\text {even }} ; t\right)-h\left(M_{\text {odd }} ; t\right)=\sum_{p, r \geq 0}(-1)^{p} \cdot t^{r} \cdot \operatorname{dim} M_{p}[r] .
$$

Let $F=\bigoplus_{r>0} F[r]$ be a graded super-algebra with $R=F[0] \subset F_{\text {even }}$. Set $F_{+}=\bigoplus_{r>0} F[r]$. We define $[F, F]_{\text {super }}$, the super-commutator space, to be the linear span of elements of the form $a b-(-1)^{|a| \cdot|b|} b a$, where $a, b$ run over even and odd elements of $F$, and we write $|x|=0$ if $x \in F_{\text {even }}$, resp. $|x|=1$ if $x \in F_{\text {odd }}$. Observe that $[F, F]_{\text {super }} \subset F_{+}$, since $R$ is a commutative algebra.

We extend the notation from (1.1.2) to the super-algebra setting and put, cf. (3.5.1),

$$
\mathcal{O}(F):=\operatorname{Sym}\left(F_{+} /[F, F]_{\text {super }}\right) .
$$

The weight, resp. homological, grading on $F$ descends to the super-commutator quotient and makes $\mathcal{O}(F)$ a super-commutative graded, or bigraded, super-algebra, $\mathcal{O}(F)=\bigoplus_{r>0} \mathcal{O}(F)[r]$, with the corresponding homological, resp. weight, gradings.

An ordinary algebra $A$ may be treated as a super-algebra with $A_{\text {odd }}=0$. In this case, formula (3.5.3) reduces to formula (1.1.2), i.e., we have $\mathcal{O}(A)=$ $\operatorname{Sym}\left(A_{+} /[A, A]\right)$.

The results of previous sections can be generalized to the case where each of the $R$-bimodules in the $(V, L)$-datum is a super-vector space. Thus, in such a case, we have $V=V_{\text {even }} \oplus V_{\text {odd }}, L=L_{\text {even }} \oplus L_{\text {odd }}$ and, moreover, the imbedding $j$ : $L \hookrightarrow T_{R}^{+} V$ respects parity, i.e., is a morphism of super-vector spaces. Then, we define the super-space $L^{\circ}:=j^{-1}\left(\left[T_{R} V, T_{R} V\right]_{\text {super }}\right)$, and set $m_{r}=\operatorname{dim}\left(L^{\circ}\right)_{\text {even }}[r]-$ $\operatorname{dim}\left(L^{\circ}\right)_{\text {odd }}[r]$.

The theory of representation schemes in the supercase is parallel to the even case, except that these schemes are now not ordinary affine schemes but rather affine superschemes (so the rings $\mathbb{C}\left[\operatorname{Rep}_{\mathbf{d}} A\right]$ are supercommutative). The notion of NCCI algebra is defined analogously to the even case. The notion of RCI 
algebra cannot be based on a dimension equality like (2.2.6), since dimension of singular super-schemes is ill-defined, in general. Instead, one defines RCI algebras by requiring that the map $p_{\mathbf{d}}$ in diagram (3.3.4) be flat which, in the even case, is equivalent to (2.2.6) by Lemma 2.3.4.

3.6. Karoubi-de Rham complex and cyclic homology. We write $\overline{H C}$. $(A)$ for reduced cyclic homology of an $R$-algebra $A$. We will also use reduced Hochschild homology of $A$, to be denoted $\overline{H H}$. $(A)$. For an augmented $R$-algebra $A$, one has $\overline{H H}_{0}(A)=\overline{H C}_{0}(A)=A /(R+[A, A])$, and $H H_{p}(A)=\overline{H H}_{p}(A)$, for all $p>0$.

Let $\Omega^{\bullet} A:=T_{A}\left(\Omega^{1} A\right)$ be the DGA of noncommutative differential forms, see [CQ]. The reduced Karoubi-de Rham complex of $A$ is defined as the supercommutator quotient

$$
\overline{\mathrm{DR}} A:=\Omega^{\bullet} A /\left(R+\left[\Omega^{\bullet} A, \Omega^{\bullet} A\right]_{\text {super }}\right) .
$$

The natural differential $\Omega^{\bullet} A \rightarrow \Omega^{\bullet+1} A$ descends to a de Rham differential on $\overline{\mathrm{DR}} A$.

Further, according to [Gi2], there is a canonical isomorphism

$$
\overline{H H} \cdot(A) \cong \operatorname{Ker}\left[\imath: \overline{\mathrm{DR}}^{\bullet} A \rightarrow \Omega^{\bullet-1} A\right],
$$

where $\imath$ is a certain canonical map that anti-commutes with the differentials. The de Rham differential gets transported, via the above isomorphism, to a natural differential $d_{H}: \overline{H H} .(A) \rightarrow \overline{H H}_{\bullet+1}(A)$ which is essentially (up to a nonzero constant factor) induced by Connes' differential $B$.

Lemma 3.6.1. Let $A$ be an $R$-algebra with vanishing reduced de Rham homology, i.e. such that $H \cdot\left(\overline{\mathrm{DR}} A, d_{D R}\right)=0$. Then, the following complex is an exact sequence

$$
0 \longrightarrow R \longrightarrow H H_{0}(A) \stackrel{d_{H}}{\longrightarrow} H H_{1}(A) \stackrel{d_{H}}{\longrightarrow} H H_{2}(A) \stackrel{d_{H}}{\longrightarrow} H H_{3}(A) \longrightarrow \ldots
$$

Furthermore, there are isomorphisms

$$
\overline{H C}_{j}(A) \cong \operatorname{Im}\left[B: H H_{j}(A) \rightarrow H H_{j+1}(A)\right], \quad \forall j \geq 0 .
$$

If, in addition, Hochschild homology groups vanish in all degrees $j>m$, for some integer $m>1$, then we deduce $\overline{H C}_{m-1}(A) \cong H H_{m}(A)$, and $\overline{H C}_{j}(A)=0$ for all $j \geq m$.

Proof. There is a standard exact sequence of reduced homology groups, see [Lo, Thm. 2.6.7]:

$$
0 \rightarrow H_{j}(\overline{\mathrm{DR}} A) \rightarrow \overline{H C}_{j}(A) \stackrel{B}{\longrightarrow} \overline{H H}_{j+1}(A) .
$$


It follows that Connes' differential $B$ is injective, for any algebra with vanishing reduced de Rham homology.

Next, we use Connes' long exact sequence, see [Lo, §2.2.13],

$$
\ldots \rightarrow \overline{H C}_{j+1}(A) \rightarrow \overline{H C}_{j-1}(A) \stackrel{B}{\longrightarrow} \overline{H H}_{j}(A) \rightarrow \overline{H C}_{j}(A) \rightarrow \ldots
$$

From the injectivity of $B$, we deduce that this long exact sequence breaks up into short exact sequences of the form

$$
0 \longrightarrow \overline{H C}_{j-1}(A) \stackrel{B}{\longrightarrow} \overline{H H}_{j}(A) \longrightarrow \overline{H C}_{j}(A) \longrightarrow 0 .
$$

Splicing all these short exact sequences together yields a long exact sequence as in (3.6.2). It is easy to verify that the maps in the two sequences are proportional, up to nonzero constant factors. Hence, the sequence in (3.6.2) is exact.

To complete the proof, assume that $H H_{j}(A)=0$ for all $j>m>1$. Then, $\overline{H H}_{j}(A)=0$, and we deduce that $\overline{H C}_{k}(A)=0$ for all $k \geq m$, due to injectivity of $B$. Hence, $\overline{H C}_{m+1}(A)=\overline{H C}_{m}(A)=0$. Now, using the short exact sequence (3.6.4) for $j=m$, we get an isomorphism $\overline{H C}_{m-1}(A) \stackrel{\sim}{\rightarrow} \overline{H H}_{m}(A)=H H_{m}(A)$, and the Lemma follows.

3.7. Cyclic Koszul complex. From now on, we fix a minimal $(V, L)$-datum. We define the cyclic Koszul complex of the algebra $A=T_{R} V /(L)$ to be the super-commutator quotient

$$
K^{\mathrm{cyc}} A:=K \cdot A /[K \cdot A, K \cdot A]_{\mathrm{super}},
$$

This is a bigraded vector space, $K_{\cdot}^{\text {cyc }} A=\bigoplus_{p, r \geq 0}\left(K_{p}^{\text {cyc }} A\right)[r]$, and the Koszul differential on $K . A$ descends to a well-defined differential $d_{K}:\left(K_{\cdot}^{\text {cyc }} A\right)[r] \rightarrow$ $\left(K_{-1}^{\mathrm{cyc}} A\right)[r]$. Furthermore, we clearly have a graded space isomorphism $H_{0}\left(K_{\cdot}^{\mathrm{cyc}} A, d_{K}\right)=A /[A, A]$.

Proposition 3.7.1. Let $A=T_{R} V /(L)$ be an NCCI algebra. Then, we have

$$
H_{1}\left(K_{\bullet}^{\mathrm{cyc}} A, d\right) \cong H H_{2}(A), \quad \text { and } \quad H_{j}\left(K_{\bullet}^{\mathrm{cyc}} A, d\right)=0, \quad \forall j>1 .
$$

Furthermore, for Hilbert series one has the formulas

$$
h(\mathcal{O}(K . A))=\zeta(V, L), \quad \text { and } \quad h(\mathcal{O}(A))=\zeta(V, L) \cdot h\left(\operatorname{Sym} H H_{2}(A)\right) .
$$

Proof. If $A$ is an NCCI algebra, then the DG algebra $K . A$ provides a free resolution of $A$, by Theorem 3.2.4(5). Thus, according to the definition of cyclic homology, the complex $\left(\left(K_{\bullet}^{\text {cyc }} A\right) / R, d_{K}\right)$ computes reduced cyclic homology of the algebra $A$. 
Now, we have that $A=\bigoplus_{r \geq 0} A[r]$ is a nonnegatively graded algebra. It follows by Poincaré lemma that the algebras $A$ and $A[0]$ have the same Karoubide Rham homology. Clearly, all Karoubi-de Rham homology groups of the algebra $A[0]=R$ vanish. Moreover, since $A$ is a NCCI algebra, we have $H H_{j}(A)=0$, for all $j>2$. Thus, by Lemma 3.6.1, we conclude that $\overline{H C}_{j}(A)=0$, for all $j>1$ and, moreover, there is an isomorphism $\overline{H C}_{1}(A) \cong H H_{2}(A)$.

Now, view the noncommutative Koszul complex $K . A$ as a free graded superalgebra, $T_{R}(V \oplus L)$, such that the vector space $V$ is even and the vector space $L$ is odd. For such a free super-algebra, the corresponding Hilbert series of $\mathcal{O}\left(T_{R}(V \oplus\right.$ $L)$ ) can be computed purely combinatorially; in accordance with formula (2.2.9) for a free (super)-algebra, one finds $h\left(\mathcal{O}\left(T_{R}(V \oplus L)\right)\right)=\zeta(V, L)$, cf. [St]. This yields the first formula in (3.7.3).

Alternatively, the same formula may be deduced from general results of $\S 4.2$ below, applied to the super-algebra $T_{R}(V \oplus L)$. Specifically, since any free superalgebra is clearly RCI, the above formula for the Hilbert series of $\mathcal{O}\left(T_{R}(V \oplus L)\right)$ is a special case of equation (4.2.4). The proof of that equation is independent of the intervening material.

Next, given a $\mathbb{Z}_{+}$-graded super-vector space $M$, write $\llbracket M \rrbracket:=\llbracket M_{\text {even }} \rrbracket-$ $\llbracket M_{\text {odd }} \rrbracket$ for the class of $M$ in the Grothendieck group of $\mathbb{Z}_{+}$-graded super-vector spaces. Applying the Euler-Poincaré principle to the complex $K^{\mathrm{cyc}} A$ and using (3.7.2), we deduce

$$
\llbracket K_{\bullet}^{\mathrm{cyc}} A \rrbracket=\llbracket H_{0}\left(K_{\bullet}^{\mathrm{cyc}} A\right) \rrbracket-\llbracket H_{1}\left(K_{\bullet}^{\mathrm{cyc}} A\right) \rrbracket=\llbracket A /[A, A] \rrbracket-\llbracket H H_{2}(A) \rrbracket .
$$

Hence, for the corresponding symmetric algebras, we find

$$
h(\mathcal{O}(A))=h\left(\operatorname{Sym} \frac{A_{+}}{[A, A]}\right)=h\left(\operatorname{Sym}\left(K_{\bullet^{\mathrm{cyc}}} A\right)_{+}\right) \cdot h\left(\operatorname{Sym} H H_{2}(A)\right) .
$$

The last equation, combined with the first formula in (3.7.3), implies the second formula in (3.7.3) since we have $\mathcal{O}(K \cdot A)=\operatorname{Sym}\left(\left(K_{\bullet}^{\mathrm{cyc}} A\right)_{+}\right)$, by definition.

Remark 3.7.4. One may use the standard identity $h(\operatorname{Sym} M) \cdot h(\wedge M)=1$, where $\wedge M$ is viewed as a super-algebra, to rewrite the second formula in (3.7.3) in the following form, more compatible with (2.2.9)(ii):

$$
h(\mathcal{O}(K . A))=\zeta(V, L) / h\left(\wedge H H_{2}(A)\right) .
$$

The assignment $\ell \mapsto 0 \oplus \ell$ gives an imbedding $L \hookrightarrow V \oplus L=T_{R}^{1}(V \oplus L)=K_{1} A$. Therefore, the vector space $L^{\circ} \subset L$, see (2.2.2), may be viewed as a subspace of $K_{1} A$. By definition of $L^{\circ}$ and of the differential on $K . A$, we have $d_{K}\left(L^{\circ}\right) \subset\left[T_{R} V, T_{R} V\right] \subset[K . A, K . A]_{\text {super }}$. Therefore, the image of $L^{\circ}$ in $K_{\cdot}^{\text {cyc }} A$ is annihilated by the differential in the cyclic Koszul complex. Thus, we obtain 
a canonical linear map

$$
L^{\circ} \rightarrow H_{1}\left(K_{\bullet}^{\mathrm{cyc}} A, d_{K}\right) .
$$

Lemma 3.7.6. The map (3.7.5) is injective. Furthermore, we have

$$
H_{j}\left(K_{\bullet}^{\mathrm{cyc}} A / L^{\circ}, d_{K}\right)=0, \forall j>0 \quad \Longleftrightarrow \quad H_{j}\left(K_{\bullet}^{\mathrm{cyc}} A, d_{K}\right)= \begin{cases}L^{\circ} & \text { if } j=1 \\ 0 & \text { if } j>1 .\end{cases}
$$

Proof. Both claims follow from the fact that $L^{\circ} \cap \operatorname{Im}\left(d_{K}\right)=0$, which is easily verified.

The second important result of this paper, to be proved in $\S 4$, reads

Theorem 3.7.7. Fix a minimal $(V, L)$-datum and set $A=T_{R} V /(L)$. Then, the following conditions (i) and (ii) are equivalent:

(i) The algebra A is NCCI and the map (3.7.5) is a bijection.

(ii) The algebra $A$ is an asymptotic RCI, cf. Definition 2.4.8.

Furthermore, the above conditions imply that $\mathrm{HH}_{2}(A) \cong L^{\circ}$, and one has

$$
h(\mathcal{O}(A))=\zeta(V, L) / \lambda\left(L^{\circ}\right), \quad \text { and } \quad h\left(\operatorname{Sym} H H_{1}(A)\right)=\zeta(V, L) / \lambda\left(L^{\circ}\right)^{2} .
$$

The above formulas for Hilbert series are identical to those in (2.2.9). Since any RCI algebra is an asymptotic RCI, we see that Theorem 3.7.7 implies Theorem 2.2.7 (the long exact sequence in (2.2.8) follows from that of Lemma (3.6.1)).

\section{The tRACE MAP}

4.1. Evaluation homomorphism. Fix an $R$ super-algebra $A$ and a dimension vector $\mathbf{d}$. Thus, one has the super-scheme $\operatorname{Rep}_{\mathbf{d}} A$.

Recall the notation $\mathbf{E}_{\mathbf{d}}=\operatorname{End}_{\mathbb{C}}\left(\bigoplus_{i \in I} \mathbb{C}^{d_{i}}\right)$, and write $\mathbf{E}_{\mathbf{d}} \otimes \mathbb{C}\left[\operatorname{Rep}_{\mathbf{d}} A\right]$, a tensor product of two associative algebras. The group $G_{\mathbf{d}}$ acts naturally on each tensor factor and we let $\left(\mathbf{E}_{\mathbf{d}} \otimes \mathbb{C}\left[\operatorname{Rep}_{\mathbf{d}} A\right]\right)^{G_{\mathbf{d}}}$ denote the subalgebra of $G_{\mathbf{d}}$-invariants with respect to the diagonal action.

To each element $a \in A$, one associates the function $\widehat{a}: \operatorname{Rep}_{\mathbf{d}} A \rightarrow \mathbf{E}_{\mathbf{d}}, \rho \mapsto$ $\widehat{a}(\rho):=\rho(a)$. The assignment $a \mapsto \widehat{a}$ clearly gives an algebra homomorphism, called evaluation map,

$$
\mathrm{ev}_{\mathbf{d}}: A \longrightarrow\left(\mathbf{E}_{\mathbf{d}} \otimes \mathbb{C}\left[\operatorname{Rep}_{\mathbf{d}} A\right]\right)^{G_{\mathbf{d}}}, \quad a \mapsto \widehat{a} .
$$

Further, we have the (super)-trace map

$$
\mathbf{E}_{\mathbf{d}} \otimes \mathbb{C}\left[\operatorname{Rep}_{\mathbf{d}} A\right] \stackrel{\operatorname{Tr} \otimes \operatorname{Id}}{\longrightarrow} \mathbb{C} \otimes \mathbb{C}\left[\operatorname{Rep}_{\mathbf{d}} A\right]=\mathbb{C}\left[\operatorname{Rep}_{\mathbf{d}} A\right] .
$$


This map clearly vanishes on (super)-commutators. Therefore, the composite of the homomorphism (4.1.1) with the trace map above descends to the (super)commutator quotient space of the algebra $A$. Thus, we obtain a well defined linear map $A /[A, A]_{\text {super }} \rightarrow \mathbb{C}\left[\operatorname{Rep}_{\mathbf{d}}\right]^{G_{\mathbf{d}}}, a \mapsto \operatorname{Tr}(\widehat{a})$.

Next, we assume that there is an augmentation, $A \rightarrow R$, with augmentation ideal $A_{+}$. The linear map $a \mapsto \operatorname{Tr}(\widehat{a})$ may be uniquely extended, by multiplicativity, to a super-algebra homomorphism

$$
\begin{aligned}
& \quad \operatorname{Tr}_{\mathbf{d}}: \mathcal{O}(A)=\operatorname{Sym}\left(A_{+} /[A, A]_{\text {super }}\right) \longrightarrow \mathbb{C}\left[\operatorname{Rep}_{\mathbf{d}}\right]^{G_{\mathbf{d}}}, \\
& a_{1} \& \ldots \& a_{m} \mapsto \operatorname{Tr}\left(\widehat{a}_{1}\right) \cdot \ldots \cdot \operatorname{Tr}\left(\widehat{a}_{m}\right), \quad \forall a_{1} \& \ldots \& a_{m} \in \operatorname{Sym}^{m}\left(A_{+} /[A, A]_{\text {super }}\right) .
\end{aligned}
$$

Proposition 4.1.3. The map $\operatorname{Tr}_{\mathbf{d}}$ is surjective.

Proof. The claim follows from Weyl's fundamental theorem of invariant theory; the ring of invariants of a collection of tensors is generated by various contractions of these tensors, cf. [LBP].

As has been noticed in [Gi1], the map $\operatorname{Tr}_{\mathbf{d}}$ becomes 'asymptotically bijective', in a sense, as $\mathbf{d} \rightarrow \infty$. One way to make this heuristic idea precise is to consider the case of algebras equipped with an additional weight grading, as we are going to do below.

4.2. Trace map for graded algebras. Fix a $(V, L)$-datum of graded $R$ superbimodules $V=V_{\text {even }} \oplus V_{\text {odd }}, L=L_{\text {even }} \oplus L_{\text {odd }}$, and set $A=T_{R} V /(L)$.

For any dimension vector $\mathbf{d}$, the grading on $V$ gives rise to a weight grading on the super-algebra $\mathbb{C}\left[\operatorname{Rep}_{\mathbf{d}} A\right]^{G_{\mathbf{d}}}$, cf. (2.3.7).

The map $\mathbf{T r}_{\mathbf{d}}$ in Proposition 4.1.3 clearly respects the gradings.

Definition 4.2.1. Assume that, for each dimension vector $\mathbf{d}$, we are given a pair of graded vector spaces, $M_{\mathbf{d}}=\bigoplus_{r \geq 0} M_{\mathbf{d}}[r]$ and $N_{\mathbf{d}}=\bigoplus_{r \geq 0} N_{\mathbf{d}}[r]$, and a linear map $f_{\mathbf{d}}: M_{\mathbf{d}} \rightarrow N_{\mathbf{d}}$ that respects the gradings.

We say that the maps $f_{\mathbf{d}}$ are asymptotically bijective as $\mathbf{d} \rightarrow \infty$ provided, for any $r=1,2, \ldots$, there exists a positive integer $n(r) \gg 0$ such that the map

$$
f_{\mathbf{d}}: \bigoplus_{s \leq r} M_{\mathbf{d}}[s] \longrightarrow \bigoplus_{s \leq r} N_{\mathbf{d}}[s]
$$

is a bijection for all $\mathbf{d} \succ n(r)$.

Proposition 11.1.1 from [CBEG] yields the following result

Proposition 4.2.2 (Stabilization). The maps $\operatorname{Tr}_{\mathbf{d}}$ are asymptotically bijective. 
Hence, for the Hilbert series $h\left(\mathbb{C}\left[\operatorname{Rep}_{\mathbf{d}} A\right]^{G_{\mathbf{d}}}\right)$, we deduce

Corollary 4.2.3. With the notation used in Corollary 2.4.9, we have

$$
h(\mathcal{O}(A))=\lim _{\mathbf{d} \rightarrow \infty} h\left(\mathbb{C}\left[\operatorname{Rep}_{\mathbf{d}} A\right]^{G_{\mathbf{d}}}\right) .
$$

We combine the equation of Corollary 4.2 .3 with the formula for the asymptotics of the matrix integral studied in $\S 2.4$. We conclude that, for any asymptotic RCI super-algebra $A=\bigoplus_{r \geq 0} A_{p}[r]$, one has

$$
h(\mathcal{O}(A)) \stackrel{\text { Coroll. 4.2.3 }}{=} \lim _{\mathbf{d} \rightarrow \infty} h\left(\mathbb{C}\left[\operatorname{Rep}_{\mathbf{d}} A\right]^{G_{\mathbf{d}}}\right) \stackrel{\text { Prop. 2.4.9 }}{=} \zeta(V, L) / \lambda\left(L^{\circ}\right) .
$$

This proves the formula (as well as its super-analogue) for the Hilbert series of $\mathcal{O}(A)$ stated in Theorem 2.2.7 and in Theorem 3.7.7.

Remark 4.2.5. Any free tensor super-algebra of the form $A=T_{R} V$ is clearly an RCI algebra, associated with the $(V, L)$-datum such that $L=0$. In this special case, the vector space $A /[A, A]_{\text {super }}$ has a basis of cyclic words (with the understanding that the cyclic word $w^{2 m}$ is equal to zero, for any word $w$ of odd homological degree and any $m \geq 1$ ), and there is an alternative purely algebraic proof of formula (4.2.4), which does not use matrix integrals and holds over fields of any characteristic, cf. [St].

Example 4.2.6. Let $A=\mathbb{C}[x]$, where $x$ is odd; so the algebra $A$ has no relations. In this case $[A, A]_{\text {super }}$ is spanned by $x^{2}, x^{4}, \ldots$, so $A_{+} /[A, A]_{\text {super }}$ has basis $x, x^{3}, x^{5}, .$. and is purely odd. Thus $\mathcal{O}(A)=\Lambda\left(x, x^{3}, \ldots\right)$, and we easily find

$$
h(\mathcal{O}(A) ; t)=(1-t)\left(1-t^{3}\right)\left(1-t^{5}\right) \cdot \ldots
$$

On the other hand, the super-version of the formula from Theorem 2.2.7, equivalently, formula (4.2.4), claims that

$$
h(\mathcal{O}(A) ; t)=\left[(1+t)\left(1+t^{2}\right)\left(1+t^{3}\right) \cdot \ldots\right]^{-1} .
$$

Thus we deduce the following well known classical identity

$$
(1-t)\left(1-t^{3}\right)\left(1-t^{5}\right) \cdot \ldots=\left[(1+t)\left(1+t^{2}\right)\left(1+t^{3}\right) \cdot \ldots\right]^{-1} .
$$

4.3. Adding dummy variables. The technique of 'dummy variables' exploited below is not new, it has been successfully applied in similar circumstances by C. Procesi, and others, cf. [Pr].

Given a finite dimensional $R$-bimodule $V$, the vector space $V^{*}=\operatorname{Hom}_{\mathbb{C}}(V, \mathbb{C})$ comes equipped with a natural $R$-bimodule structure, that is, with a bigrading $V^{*}=\bigoplus_{i, j \in I} V_{i j}^{*}$ such that $V_{i j}^{*}=\left(V_{j i}\right)^{*}$. 
Now, fix a $(V, L)$-datum and put $V^{b}:=V \oplus V^{*} \oplus L^{*}$, where $V^{b}$ is viewed as a $R$-super-bimodule such that $\left(V^{b}\right)_{\text {even }}:=V \oplus V^{*}$ and $\left(V^{b}\right)_{\text {odd }}:=L^{*}$. The assignment $\ell \mapsto j(\ell) \in T_{R} V \subset T_{R}\left(V^{b}\right)$ gives an $R$-super-bimodule imbedding $j^{b}: L \hookrightarrow T_{R}\left(V^{b}\right)$. We put $A^{b}:=T_{R}\left(V^{b}\right) /(L)$, where $(L)$ stands for the two-sided ideal generated by the image of $j^{b}$.

Lemma 4.3.1. The complex $\left(K_{\cdot}^{\mathrm{cyc}} A^{b}\right) / L^{\circ}$ is acyclic in positive homological degrees if and only if each of the two complexes $K . A$ and $K_{\cdot}^{\mathrm{cyc}} A / L^{\circ}$ is acyclic in positive homological degrees.

Proof. An element $m$ of an $R$ super-bimodule $M$ is called homogeneous if $x \in$ $M_{\text {even }}$, in which case we set $|x|=0$, or $x \in M_{\text {odd }}$, in which case we set $|x|=1$. Given an integer $k \geq 1$, let $M_{\text {anticyc }}^{\otimes k}$ denote a quotient of $T_{R}^{k} M$ modulo all relations of the form

$m_{1} \otimes \ldots \otimes m_{k}=(-1)^{q} \cdot m_{k} \otimes m_{1} \ldots \otimes m_{k-1}, \quad r \cdot m_{1} \otimes \ldots \otimes m_{k}=m_{1} \otimes \ldots \otimes m_{k} \cdot r$, where $r \in R$ and $m_{1}, \ldots, m_{k} \in M$ are homogeneous elements, and where we put $q:=\left|m_{k}\right| \cdot\left(\left|m_{1}\right|+\ldots+\left|m_{k-1}\right|\right)$.

Now, let $B=R \oplus B_{+}$and $C=R \oplus C_{+}$be a pair of graded augmented $R$ super-algebras and let $B *_{R} C$ denote their free product over $R$. Clearly, we have

$\left(\left(4 B 3 \mathrm{Q}_{R} C\right) /\left[B *_{R} C, B *_{R} C\right]=B /[B, B] \bigoplus C /[C, C] \bigoplus\left(B_{+} \otimes_{R} C_{+}\right)\right.$

$$
\bigoplus\left(B_{+} \otimes_{R} C_{+}\right)_{\text {anticyc }}^{\otimes 2} \bigoplus\left(B_{+} \otimes_{R} C_{+}\right)_{\text {anticyc }}^{\otimes 3} \bigoplus \ldots,
$$

where in the super-case the commutators are to be replaced by super-commutators.

We apply this in the special case $B:=T_{R}\left(V^{*} \oplus L^{*}\right)$ and $C:=T_{R}(V \oplus L)$. We have $K . A^{b}=T\left(V \oplus V^{*} \oplus L \oplus L^{*}\right)=B *_{R} C$, where the factor $B$ sits in homological degree 0 . Therefore, from (4.3.2) we obtain

$K_{\cdot}^{\mathrm{cyc}} A^{\mathrm{b}}=\frac{B}{[B, B]} \bigoplus K_{\cdot}^{\mathrm{cyc}} A \bigoplus\left(B_{+} \otimes_{R}(K \cdot A)_{+}\right) \bigoplus\left(B_{+} \otimes_{R}(K \cdot A)_{+}\right)_{\text {anticyc }}^{\otimes 2} \ldots$,

where $B$ is viewed as a DG super-algebra with zero differential concentrated in homological degree zero.

Hence, for homology we get

$$
H \cdot\left(\left(K_{\bullet}^{\mathrm{cyc}} A^{b}\right) / L^{\circ}\right)=\frac{B}{[B, B]} \bigoplus H \cdot\left(K_{\cdot}^{\mathrm{cyc}} A / L^{\circ}\right) \bigoplus B_{+} \otimes_{R} H \cdot\left((K \cdot A)_{+}\right) \bigoplus \ldots
$$

We see that that the homology in the RHS is concentrated in degree 0 if and only if each of the two complexes $K_{{ }^{\mathrm{cyc}}} A / L^{\circ}$ and $K . A$ has vanishing homology in degrees $>0$.

Remark 4.3.3. The above Lemma may be seen as an instance of cyclic Kunneth formula for free products. 
Definition 4.3.4. Let $M^{\mathbf{d}}=\bigoplus_{p \in \mathbb{Z}, r \geq 0} M_{p}^{\mathbf{d}}[r]$, be a collection of bigraded complexes (one for each $\mathbf{d} \in \mathbb{Z}_{+}^{I}$ ), with differentials $d=d^{\mathbf{d}}: M_{\bullet}^{\mathbf{d}}[r] \rightarrow M_{\bullet_{-1}}^{\mathbf{d}}[r]$.

We say that the complexes $\left(M^{\mathrm{d}}, d\right)$ are asymptotically acyclic in positive homological degrees as $\mathbf{d} \rightarrow \infty$ if, for any $r, N \geq 1$, there exists a dimension vector $\mathbf{d}=\mathbf{d}(r, N) \succ N$ such that one has $H_{k}\left(\bigoplus_{s \leq r} M_{\bullet}^{\mathbf{d}}[s]\right)=0$ for all $k>0$.

Proposition 4.3.5. For the graded super-algebra $A=T_{R} V /(L)$ associated to a $(V, L)$-datum, the following are equivalent:

(i) The complexes $\left(\mathbf{K} .\left(\operatorname{Rep}_{\mathbf{d}} A^{b}\right)^{G_{\mathbf{d}}}, d_{K}\right)$, of $G_{\mathbf{d}}$-invariants, are asymptotically acyclic in positive homological degrees as $\mathbf{d} \rightarrow \infty$.

(ii) The complexes $\left(\mathbf{K} .\left(\operatorname{Rep}_{\mathbf{d}^{\prime}} A\right), d_{K}\right)$ are asymptotically acyclic in positive homological degrees as $\mathbf{d}^{\prime} \rightarrow \infty$.

Proof. The graded super-algebra $A^{b}$ is clearly isomorphic to $A *_{R} T_{R}\left(V^{*} \oplus L^{*}\right)$, a free product (over $R$ ) of $A$, and the tensor algebra $T_{R}\left(V^{*} \oplus L^{*}\right)$.

For any dimension vector $\mathbf{d}$, we have $\operatorname{Rep}_{\mathbf{d}} T_{R}\left(V^{*} \oplus L^{*}\right)=\mathbb{V}_{\mathbf{d}}^{*} \oplus \mathbb{L}_{\mathbf{d}}^{*}$, where $\mathbb{V}_{\mathbf{d}}^{*} \oplus \mathbb{L}_{\mathbf{d}}^{*}$ is the bigraded vector space dual to $\mathbb{V}_{\mathbf{d}} \oplus \mathbb{L}_{\mathbf{d}}$. Therefore, one has a canonical isomorphism

$$
\operatorname{Rep}_{\mathbf{d}} A^{b} \cong \operatorname{Rep}_{\mathbf{d}} A \times \operatorname{Rep}_{\mathbf{d}} T_{R}\left(V^{*} \oplus L^{*}\right)=\operatorname{Rep}_{\mathbf{d}} A \times \mathbb{V}_{\mathbf{d}}^{*} \times \mathbb{L}_{\mathbf{d}}^{*} \subset \mathbb{V} \times \mathbb{V}_{\mathbf{d}}^{*} \times \mathbb{L}_{\mathbf{d}}^{*}
$$

where all the sets above are viewed as super-schemes. For the corresponding Koszul complexes, this gives the following $G_{\mathbf{d}} \times \mathbb{C}^{\times}$-equavariant DG algebra isomorphism

$$
\mathbf{K} .\left(\operatorname{Rep}_{\mathbf{d}} A^{b}\right) \cong \mathbf{K} \cdot\left(\operatorname{Rep}_{\mathbf{d}} A \times \mathbb{V}_{\mathbf{d}}^{*} \times \mathbb{L}_{\mathbf{d}}^{*}\right) \cong \mathbf{K} \cdot\left(\operatorname{Rep}_{\mathbf{d}} A\right) \otimes\left(\mathbb{C}\left[\mathbb{V}_{\mathbf{d}}^{*}\right] \otimes \Lambda \mathbb{L}_{\mathbf{d}}^{*}\right),
$$

where the rightmost tensor factor $\mathbb{C}\left[\mathbb{V}_{\mathbf{d}}^{*}\right] \otimes \Lambda \mathbb{L}_{\mathbf{d}}^{*}$ is a DG super-algebra equipped with zero differential and concentrated in homological degree zero.

Now fix integers $r, N$ and assume that the complex $\left(\bigoplus_{s \leq r} \mathbf{K} .\left(\operatorname{Rep}_{\mathbf{d}} A\right)[s], d_{K}\right)$ is acyclic in positive homological degrees, for some $\mathbf{d} \succ N$. We see from (4.3.6) that a similar statement holds for the complex $\mathbf{K}$. $\left(\operatorname{Rep}_{\mathbf{d}} A^{\mathrm{b}}\right)$ as well. It follows that the same holds for the subcomplex K. $\left(\operatorname{Rep}_{\mathbf{d}} A^{b}\right)^{G_{\mathbf{d}}}$, of $G_{\mathbf{d}}$-invariants. This proves the implication (i) $\Rightarrow$ (ii) of the Proposition.

To prove the opposite implication, we use complete reducibility of finite dimensional $G_{\mathrm{d}}$-representations. Specifically, given a (finite dimensional) rational $G_{\mathbf{d}}$-representation $U$ and an irreducible $G_{\mathbf{d}}$-representation $M$, let $U^{M}=$ $\operatorname{Hom}_{G_{\mathbf{d}}}(M, U)$ be the $M$-multiplicity space of $U$. For any pair $U, W$, of finite dimensional $G_{\mathbf{d}}$-representations, by complete reducibility, one has a canonical direct sum decomposition of the space of $G_{\mathbf{d}^{-}}$-diagonal invariants: $(U \otimes W)^{G_{\mathbf{d}}}=$ $\bigoplus_{M \in \operatorname{Irr}\left(G_{\mathbf{d}}\right)} U^{M} \otimes W^{M^{*}}$, where $M^{*}$ stands for the contragredient representation. 
Now, the group $G_{\mathbf{d}}$ acts diagonally on the tensor product in the RHS of (4.3.6). Hence, taking $G_{\mathbf{d}}$-invariants we obtain

$$
\begin{aligned}
\mathbf{K} .\left(\operatorname{Rep}_{\mathbf{d}} A^{b}\right)^{G_{\mathbf{d}}} & =\left(\mathbf{K} .\left(\operatorname{Rep}_{\mathbf{d}} A\right) \otimes \mathbb{C}\left[\mathbb{V}_{\mathbf{d}}^{*}\right] \otimes \Lambda \mathbb{L}_{\mathbf{d}}^{*}\right)^{G_{\mathbf{d}}} \\
& =\bigoplus_{M \in \operatorname{Irr}\left(G_{\mathbf{d}}\right)} \mathbf{K} \cdot\left(\operatorname{Rep}_{\mathbf{d}} A\right)^{M} \otimes\left(\mathbb{C}\left[\mathbb{V}_{\mathbf{d}}^{*}\right] \otimes \Lambda \mathbb{L}_{\mathbf{d}}^{*}\right)^{M^{*}}
\end{aligned}
$$

Observe also that, for each irreducible $G_{\mathbf{d}}$-representation $M$, one clearly has

$$
\text { K. }\left(\operatorname{Rep}_{\mathbf{d}} A\right)^{M}=\left(\mathbb{C}\left[\mathbb{V}_{\mathbf{d}}\right] \otimes \Lambda \mathbb{L}_{\mathbf{d}}\right)^{M} \neq 0 \Longleftrightarrow\left(\mathbb{C}\left[\mathbb{V}_{\mathbf{d}}^{*}\right] \otimes \Lambda \mathbb{L}_{\mathbf{d}}^{*}\right)^{M^{*}} \neq 0
$$

To complete the proof, fix integers $r, N$, and assume that for some $\mathbf{d} \succ N$ the complex $\left(\bigoplus_{s \leq r} \mathbf{K} .\left(\operatorname{Rep}_{\mathbf{d}} A^{b}\right)[s]^{G_{\mathbf{d}}}, d_{K}\right)$ is acyclic in positive homological degrees. We deduce from the direct sum decomposition in (4.3.7) and from (4.3.8) that, increasing the values of $r$ and $\mathbf{d}$ if necessary, one can ensure that a similar statement holds for each $G_{\mathbf{d}^{-}}$-isotypic component that occurs with nonzero multiplicity in the complex $\bigoplus_{s \leq r} \mathbf{K}$. $\left(\operatorname{Rep}_{\mathbf{d}}\right)[s]$. The implication (ii) $\Rightarrow$ (i) follows.

4.4. Proof of Theorem 3.7.7. Given any $(V, L)$-datum, we may view the tensor algebra $T_{R}(V \oplus L)$ as a free bigraded super-algebra such that the vector space $V$ is even and is placed in homological degree 0 , resp. the vector space $L$ is odd and is placed in homological degree 1 (cf. Proof of Proposition 3.7.1).

We apply the general construction of the trace map $\operatorname{Tr}_{\mathbf{d}}$, cf. (4.1.2), to the free bigraded super-algebra $K \cdot A=T_{R}(V \oplus L)$. Since $\operatorname{Rep}_{\mathbf{d}}\left(T_{R}(V \oplus L)\right) \cong \mathbb{V}_{\mathbf{d}} \oplus \mathbb{L}_{\mathbf{d}}$, we obtain a bigraded super-algebra homomorphism

$$
\operatorname{Tr}_{\mathbf{d}}: \mathcal{O}(K \cdot A)=\operatorname{Sym}\left(\left(K_{\bullet}^{\mathrm{cyc}} A\right)_{+}\right) \longrightarrow\left(\mathbb{C}\left[\mathbb{V}_{\mathbf{d}}\right] \otimes \Lambda^{\cdot} \mathbb{L}_{\mathbf{d}}^{*}\right)^{G_{\mathbf{d}}} .
$$

It is straightforward to check that this map intertwines the Koszul differentials on both sides, i.e., it is a morphism of DG algebras.

Next, recall the short exact sequence from (2.3.11) and the isomorphism in (2.3.12). It is easy to see that the following diagram commutes

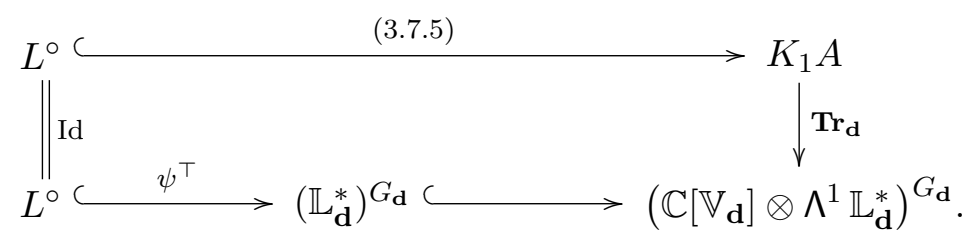

Therefore, the map $\operatorname{Tr}_{\mathbf{d}}$ descends to a well defined map

$$
\operatorname{Tr}_{\mathbf{d}}^{\circ}: \operatorname{Sym}\left(\left(K_{\bullet}^{\mathrm{cyc}} A\right)_{+} / L^{\circ}\right) \longrightarrow\left(\mathbb{C}\left[\mathbb{V}_{\mathbf{d}}\right] \otimes \Lambda^{\bullet}\left(\mathbb{L}_{\mathbf{d}}^{*} / L^{\circ}\right)\right)^{G_{\mathbf{d}}}=\mathbf{K} \cdot\left(\operatorname{Rep}_{\mathbf{d}} A\right)^{G_{\mathbf{d}}} \text {. }
$$


(here and below, we will identify the space $L^{\circ}$ with its image $\left.\psi^{\top}\left(L^{\circ}\right) \subset \mathbb{L}_{\mathbf{d}}^{*}\right)$. The map (4.4.3) is again a bigraded super-algebra homomorphism compatible with the Koszul differentials on each side.

Lemma 4.4.4. The maps $\operatorname{Tr}_{\mathbf{d}}^{\circ}$ are asymptotically bijective, cf. Definition 4.2.1.

Proof. Since any free algebra is RCI, Proposition 4.2.2 implies that the map $\mathbf{T r}_{\mathbf{d}}$ in (4.4.1) is an asymptotically bijective algebra homomorphism. By commutativity of diagram (4.4.2), this homomorphism sends the subspace $L^{\circ} \subset \mathcal{O}(K . A)$ isomorphically onto the corresponding subspace $\psi^{\top}\left(L^{\circ}\right) \subset \mathbf{K}$. $\left(\operatorname{Rep}_{\mathbf{d}} A\right)^{G_{\mathbf{d}}}$. Therefore, the map $\operatorname{Tr}_{\mathbf{d}}$ yields an asymptotic bijection between the ideal of the algebra $\mathcal{O}(K . A)$ generated by the subspace $L^{\circ}$ and the ideal of the algebra $\mathbf{K} .\left(\operatorname{Rep}_{\mathbf{d}} A\right)^{G_{\mathbf{d}}}$ generated by the subspace $\psi^{\top}\left(L^{\circ}\right)$. The induced asymptotic bijection $\mathcal{O}(K . A) /$ $\mathcal{O}(K . A) \cdot L^{\circ} \stackrel{\sim}{\rightarrow} \mathbf{K} .\left(\operatorname{Rep}_{\mathbf{d}} A\right)^{G_{\mathbf{d}}} / \mathbf{K} .\left(\operatorname{Rep}_{\mathbf{d}} A\right)^{G_{\mathbf{d}}} \cdot \psi^{\top}\left(L^{\circ}\right)$ is nothing but the map $\operatorname{Tr}_{\mathbf{d}}^{\circ}$, since we have

$$
\left(\mathbb{C}\left[\mathbb{V}_{\mathbf{d}}\right] \otimes \Lambda^{\bullet} \mathbb{L}_{\mathbf{d}}^{*}\right)^{G_{\mathbf{d}}} /\left(\mathbb{C}\left[\mathbb{V}_{\mathbf{d}}\right] \otimes \Lambda^{\bullet} \mathbb{L}_{\mathbf{d}}^{*}\right)^{G_{\mathbf{d}}} \cdot L^{\circ} \cong\left(\mathbb{C}\left[\mathbb{V}_{\mathbf{d}}\right] \otimes \Lambda^{\bullet}\left(\mathbb{L}_{\mathbf{d}}^{*} / L^{\circ}\right)\right)^{G_{\mathbf{d}}}
$$

Proof of Theorem 3.7.7. Assume first that the condition of Theorem 3.7.7(ii) holds. Thus, the complexes $\left(\mathbf{K} .\left(\operatorname{Rep}_{\mathbf{d}^{\prime}} A\right), d_{K}\right)$ are asymptotically acyclic in positive homological degrees as $\mathbf{d}^{\prime} \rightarrow \infty$, cf. Definition 4.3.4. Hence, the complexes $\left(\mathbf{K} .\left(\operatorname{Rep}_{\mathbf{d}} A^{b}\right)^{G_{\mathbf{d}}}, d_{K}\right)$, of $G_{\mathbf{d}^{-}}$invariants, are asymptotically acyclic in positive homological degrees as $\mathbf{d} \rightarrow \infty$, by Proposition 4.3.5. Applying Lemma 4.4.4 to the algebra $A^{b}$, we deduce that the complex $\left(K_{0}^{\mathrm{cyc}} A^{\mathrm{b}}\right) / L^{\circ}$ is acyclic in positive homological degrees. This implies, by Lemma 4.3.1, that each of the complexes $K . A$ and $\left(K_{\bullet}^{\mathrm{cyc}} A\right) / L^{\circ}$ is acyclic in positive homological degrees. Thus, $A$ is a NCCI, by Theorem 3.2.4(5). Now, the implication (ii) $\Rightarrow$ (i) of Theorem 3.7.7 follows from Lemma 3.7.6.

Conversely, assume condition (i) of Theorem 3.7.7 holds. From Lemma 4.3.1, we deduce that the complex $\left(K_{\cdot}^{\text {cyc }} A^{b}\right) / L^{\circ}$ is acyclic in positive homological degrees. Hence, the complexes $\left(\mathbf{K} .\left(\operatorname{Rep}_{\mathbf{d}} A^{b}\right)^{G_{\mathbf{d}}}, d_{K}\right)$, of $G_{\mathbf{d}^{-}}$invariants, are asymptotically acyclic in positive homological degrees as $\mathbf{d} \rightarrow \infty$, by Lemma 4.4.4 applied to the algebra $A^{b}$. Therefore, the complexes $\left(\mathbf{K} .\left(\operatorname{Rep}_{\mathbf{d}^{\prime}} A\right), d_{K}\right)$ are asymptotically acyclic in positive homological degrees as $\mathbf{d}^{\prime} \rightarrow \infty$, by Proposition 4.3.5. This proves the implication (i) $\Rightarrow$ (ii).

Finally, the equations for Hilbert series given in Theorem 3.7.7 follow from (3.7.3).

\section{Additional Results and examples}

5.1. Twisted free products. In this subsection, we provide a few useful ways of producing NCCI, resp. RCI algebras. 
First, let $A_{j}=T_{R} V_{j} /\left(L_{j}\right), j=1,2$, be a pair graded algebras associated with $\left(V_{i}, L_{i}\right)$-data. The free product of $A_{1}$ and $A_{2}$ over $R$ is a graded algebra

$$
A_{1} *_{R} A_{2} \cong T_{R}\left(V_{1} \oplus V_{2}\right) /\left(L_{1} \oplus L_{2}\right) \text {. }
$$

One easily finds

$$
h\left(A_{1} *_{R} A_{2}\right)=\left(h\left(A_{1}\right)^{-1}+h\left(A_{2}\right)^{-1}-\mathbf{1}\right)^{-1} .
$$

Proposition 5.1.2. (i) Let $A=T_{R} V /(L)$, and $B=T_{R} V /\left(L^{\prime}\right)$, where $L^{\prime} \subset L$ is a subbimodule. Then, if $A$ is $R C I$ then $B$ is $R C I$.

(ii) If $A_{1} *_{R} A_{2}$ is $R C I$ then both $A_{1}$ and $A_{2}$ are $R C I$.

(iii) The converse to (ii) holds if, in Definition 2.2.5, for each $N \gg 0$ one can use the same dimension $\mathbf{d}$ both for $A_{1}$ and $A_{2}$.

Proof. Observe that if an algebraic map to a vector space is flat then so is the induced map to any quotient vector space. Part (i) follows. Parts (ii)-(iii) are immediate from (2.2.6).

Assume next that one has a $(V, L)$-data and an $(L, M)$-data, that is, there are finite dimensional $\mathbb{N}$-graded $R$-bimodules $V, L, M$ and graded maps

$$
j_{L}: L \hookrightarrow T_{R}^{+} V, \text { and } j_{M}^{\prime}: M \hookrightarrow j_{M} \longrightarrow T_{R}^{+} L \stackrel{T\left(j_{L}\right)}{\longrightarrow} T_{R}^{+} V,
$$

where $T\left(j_{L}\right)$ is the algebra morphism induced by the $R$-bimodule morphism $j_{L}$. Thus, we may define a triple of graded $R$-algebras

$$
B:=T_{R} V /\left(\operatorname{Im} j_{L}\right), \quad D:=T_{R} L /\left(\operatorname{Im} j_{M}\right), \quad B \circ_{R} D:=T_{R} V /\left(\operatorname{Im} j_{M}^{\prime}\right) .
$$

There is a slightly different but equivalent interpretation of the algebra $B \circ_{R} D$. To explain it, consider two $R$-bimodule maps

$$
\begin{aligned}
j_{L M}, j_{L M}^{\prime}: L \oplus M \longrightarrow T_{R}(V \oplus L), & j_{L M}(\ell \oplus m):=j_{L}(\ell)+j_{M}(m), \\
& j_{L M}^{\prime}(\ell \oplus m):=j_{L}(\ell)-\ell+j_{M}(m) .
\end{aligned}
$$

We observe that the algebra $B *_{R} D$, a free product, is a quotient of $T_{R}(V \oplus L)$ by the two-sided ideal generated by the image of the map $j_{L M}$. Observe further that the kernel of the algebra homomorphism

$$
T_{R}(V \oplus L) \rightarrow T_{R} V /\left(\operatorname{Im} j_{M}^{\prime}\right)=B \circ_{R} D,
$$

induced by the first projection $V \oplus L \rightarrow V$, is a two-sided ideal in $T_{R}(V \oplus L)$ generated by the image of the map $j_{L M}^{\prime}$. Therefore, we get

$$
B *_{R} D=T_{R}(V \oplus L) /\left(\operatorname{Im} j_{L M}\right), \quad \text { and } \quad B \circ_{R} D=T_{R}(V \oplus L) /\left(\operatorname{Im} j_{L M}^{\prime}\right) \text {. }
$$


Proposition 5.1.6. If $B$ is an NCCI algebra then, for the Hilbert series of $B \circ_{R} D$, we have

$$
h\left(B \circ_{R} D\right)=[\mathbf{1}-h(D) \cdot(h(V)-h(L))]^{-1} \cdot h(D) .
$$

To prove the proposition, we introduce an increasing filtration on the algebra $B \circ_{R} D$ as follows. First define a grading on $T_{R}(V \oplus L)$ by assigning elements of the vector space $V \subset V \oplus L$ their natural degrees and placing the vector space $L \subset V \oplus L$ in degree zero. The increasing filtration on $T_{R}(V \oplus L)$ induced by this grading descends to an increasing filtration on the quotient algebra $B \circ_{R} D=$ $T_{R}(V \oplus L) /\left(\operatorname{Im} j_{L M}^{\prime}\right)$. Let $\operatorname{gr}\left(B \circ_{R} D\right)$ denote the corresponding associated graded algebra.

It is clear from the isomorphisms in (5.1.5) that the tautological imbedding $V \oplus L \hookrightarrow T_{R}(V \oplus L)$ induces a well defined and surjective graded algebra homomorphism

$\xi: B *_{R} D=T_{R}(V \oplus L) /\left(\operatorname{Im} j_{L M}\right) \rightarrow \operatorname{gr}\left(B \circ_{R} D\right)=\operatorname{gr}\left(T_{R}(V \oplus L) /\left(\operatorname{Im} j_{L M}^{\prime}\right)\right)$.

Lemma 5.1.8. If $B$ is an NCCI algebra then the map (5.1.7) is an isomorphism.

Proof of Lemma. Let $F=T_{R} V$, and to simplify notation write $\otimes=\otimes_{R},(-) *$ $(-)=(-) *_{R}(-)$, etc. The noncommutative Koszul complex $K \cdot(B)$ for the algebra $B$ reads

$$
\ldots \rightarrow F \otimes L \otimes F \otimes L \otimes F \rightarrow F \otimes L \otimes F \rightarrow F \rightarrow B .
$$

Taking a free product of this complex with the algebra $D$ yields a complex of the form

$$
\ldots \rightarrow(F * D) \otimes L \otimes(F * D) \otimes L \otimes(F * D) \rightarrow(F * D) \otimes L \otimes(F * D) \rightarrow F * D \rightarrow B * D .
$$

The complex $K .(B)$ provides a resolution of $B$, since $B$ is an NCCI algebra. It follows that the complex in (5.1.9) provides a resolution for the algebra $B * D$.

On the other hand, using the presentation of the algebra $B \circ D$ given in (5.1.5), one sees that the terms of the corresponding Koszul complex $K .(B \circ D)$ can be written in the form

$$
\ldots \rightarrow(F * D) \otimes L \otimes(F * D) \otimes L \otimes(F * D) \rightarrow(F * D) \otimes L \otimes(F * D) \rightarrow F * D \rightarrow B \circ D .
$$

Now, the above defined increasing filtration on $B \circ D$ gives rise to an increasing filtration on the DG algebra $K .(B \circ D)$. Furthermore, it is clear that the complex (5.1.9) is obtained by taking the associated graded of the complex (5.1.10) with respect to that filtration. Hence, it follows that, for an NCCI algebra $B$, the complex (5.1.10) is acyclic in positive homological degrees. This implies that $\xi$ is an isomorphism, and the lemma is proved. 
Proof of Proposition 5.1.6. Since $B$ is NCCI, we have $h(B)=(\mathbf{1}-h(V)+h(L))^{-1}$. Further, by Lemma 5.1.8, the graded $R$-algebras $B \circ D$ and $B * D$ have the same Hilbert series. The proposition now follows from formula (5.1.1).

5.2. We keep the notation of (5.1.3)-(5.1.4). Also put $F:=T_{R} V$, and $A:=B \circ_{R} D$ and $A^{\prime}:=B *_{R} D$. We have the sub $R$-bimodule $L^{\circ}=L \cap[F, F]$.

Let $Q:=L \cap[F, F] \cap\left(\operatorname{Im} j_{M}\right)$ be the kernel of the natural map $\eta: L^{\circ} \rightarrow D=$ $T_{R} L /\left(j_{M}\right)$.

Proposition 5.2.1. If $B$ is an asymptotic RCI algebra then one has

$$
h\left(\mathcal{O}\left(B \circ_{R} D\right) ; t\right)=\frac{h(\mathcal{O}(D) ; t)}{\lambda(Q) \cdot \prod_{s \geq 1} \operatorname{det}\left(\mathbf{1}+h\left(D ; t^{s}\right) \cdot\left[h\left(V ; t^{s}\right)-h\left(L ; t^{s}\right)\right]\right)} .
$$

Let $\bar{L}^{\circ}$ be the image of the map $\eta: L^{\circ} \rightarrow D$. We have a natural embedding $\gamma: \bar{L}^{\circ} \hookrightarrow[A, A]$. The image of $\gamma$ is contained in the degree zero part of $[A, A]$ under the filtartion on $A$, so it gives rise to an embedding $\operatorname{gr} \gamma: \bar{L}^{\circ} \hookrightarrow \operatorname{gr}([A, A])$.

Lemma 5.2.2. One has $\operatorname{gr}([A, A])=\left[A^{\prime}, A^{\prime}\right] \oplus(\operatorname{gr} \gamma)\left(\bar{L}^{\circ}\right)$.

Proof. The direct sum of the terms of the complex (5.1.10), resp. (5.1.9), gives a differential graded algebra $K_{\bullet}$, resp. $K_{\bullet}^{\prime}=K_{\bullet}(B) *_{R} D$. As we have explained earlier, the DG algebra $K$. comes equipped with the increasing filtration, such that one has gr $K_{\bullet}=K_{.}^{\prime}$. Therefore, for the corresponding super-commutator quotients, $K_{\bullet}^{\mathrm{cyc}}:=K_{\bullet} /\left[K_{\bullet}, K_{\bullet}\right]$ and $\left(K_{\bullet}^{\mathrm{cyc}}\right)^{\prime}:=K_{\bullet}^{\prime} /\left[K_{\bullet}^{\prime}, K_{\bullet}^{\prime}\right]$, respectively, we deduce gr $K_{\bullet}^{\mathrm{cyc}}=\left(K_{\bullet}^{\mathrm{cyc}}\right)^{\prime}$.

Since $B$ is an asymptotic $R C I$ algebra, the homology of the complex gr $K_{*}^{\text {cyc }}=$ $\left(K_{\cdot}^{\text {cyc }}\right)^{\prime}$ is concentrated in degrees 0 and 1 . Moreover, we have $H_{0}\left(\left(K_{\bullet}^{\text {cyc }}\right)^{\prime}\right)=$ $A^{\prime} /\left[A^{\prime}, A^{\prime}\right]$ and $H_{1}\left(\left(K_{\bullet}^{\mathrm{cyc}}\right)^{\prime}\right)=L^{\circ}$.

The increasing filtration on $K_{\cdot}^{\text {cyc }}$ gives rise to a standard spectral sequence with $E_{1}$-term $H_{\bullet}\left(\mathrm{gr} K_{.}^{\mathrm{cyc}}\right)$ that converges to the homology of $K_{\bullet}^{\text {cyc }}$. It can be seen from the analysis of the spectral sequence that this yields $H_{1}\left(K_{\bullet}\right)=Q$. We also have $H_{0}\left(K_{\bullet}\right)=A /[A, A]$. The lemma follows.

Proof of Proposition 5.2.1. By Proposition 5.2.2, to compute the Hilbert series of $\mathcal{O}(A)$ it suffices to compute the Hilbert series of $\mathcal{O}\left(A^{\prime}\right)$. To compute the latter, note that we have

$$
\frac{A_{+}^{\prime}}{\left[A^{\prime}, A^{\prime}\right]}=\frac{B_{+}}{[B, B]} \oplus \frac{D_{+}}{[D, D]} \oplus \frac{T_{R}\left(B_{+} \otimes_{R} D_{+}\right)_{+}}{\left[T_{R}\left(B_{+} \otimes_{R} D_{+}\right), T_{R}\left(B_{+} \otimes_{R} D_{+}\right)\right]} .
$$

This implies that

$$
h\left(\mathcal{O}\left(A^{\prime}\right) ; t\right)=\frac{h(\mathcal{O}(B) ; t) \cdot h(\mathcal{O}(D) ; t)}{\prod_{s \geq 1} \operatorname{det}\left(h\left(B ; t^{s}\right)+h\left(D ; t^{s}\right)-h\left(B ; t^{s}\right) \cdot h\left(D ; t^{s}\right)\right)} .
$$


Using that $B$ is an asymptotic RCI algebra, the last equation can be rewritten as

$$
h\left(\mathcal{O}\left(A^{\prime}\right) ; t\right)=\frac{1}{\lambda(Q)} \frac{h(\mathcal{O}(D) ; t)}{\prod_{s \geq 1} \operatorname{det}\left(\mathbf{1}+h\left(D ; t^{s}\right) \cdot\left[h\left(V ; t^{s}\right)-h\left(L ; t^{s}\right)\right]\right)},
$$

and the proposition follows.

5.3. Examples of RCI algebras. We begin with the example of the algebra of $q$-polynomials. Thus, we fix $q \in \mathbb{C}^{\times}$, and let $\mathbb{C}_{q}[x, y]$ be the algebra with two generators $x, y$ of degree 1 and defining relation $x y-q y x=0$.

Proposition 5.3.1. If $q$ is not a root of unity, then the algebra $\mathbb{C}_{q}[x, y]$ is $R C I$.

Remark 5.3.2. (i) If $q$ is a root of unity then the $q$-polynomial algebra $\mathbb{C}_{q}[x, y]$ is not a RCI algebra. Namely, if $n$ is the order of $q$ then $\mathbb{C}_{q}[x, y]$ has a 2-parameter family of equivalence classes of irreducible $n$-dimensional representations, which gives an $n^{2}+1$-dimensional family of representations. Taking direct sums of such representations and 1-dimensional representations, we see that $\operatorname{Rep}_{d}(A)$ has dimension bigger than expected, for any $d \geq n$.

(ii) Formulas (2.2.9)(i)-(ii) for $\mathbb{C}_{q}[x, y]$ are easy to check directly. If $q$ is a root of unity, then it is easy to see that (i) still holds but (ii) fails (which is another way to see that in this case $\mathbb{C}_{q}[x, y]$ is not $\mathrm{RCI}$ ).

Proof. We need to show that the variety $R_{n}$ of $n$-dimensional representations of the algebra $\mathbb{C}_{q}[x, y]$ has dimension $n^{2}$. To do so, consider the subvariety $R_{n}^{0}$ of $R_{n}$ consisting of representations on which $x$ is a nilpotent matrix. Since $x$ in this case is conjugate to $q x$, the dimension of the space of solutions $y$ of the equation $x y=q y x$ is the same as the dimension of the centralizer of $x$. Therefore, for each nilpotent conjugacy class $C$ (corresponding to the matrix $x$ ), the dimension of the set $\{(x, y) \mid x y=q y x, x \in C\}$ equals $n^{2}$. Thus, we get $\operatorname{dim} R_{n}^{0}=n^{2}$.

Any representation $V \in R_{n}$, of $\mathbb{C}_{q}[x, y]$, has a canonical direct sum decomposition into Fitting components $V=V_{1} \oplus V_{2}$, such that the endomorphism $\left.x\right|_{V_{1}}$ is nilpotent and the endomorphism $\left.x\right|_{V_{2}}$ is invertible (and then $y$ is nilpotent because $q$ is not a root of unity). Let $\operatorname{dim} V_{1}=n_{1}$, and $\operatorname{dim} V_{2}=n_{2}$. Then there are $\left(n_{i}\right)^{2}$ parameters for $V_{i}$, and an additional amount of $2 n_{1} n_{2}$ parameters for the choice of the vector space decomposition $V=V_{1} \oplus V_{2}$. Thus the total dimension is $\left(n_{1}+n_{2}\right)^{2}=n^{2}$, and we are done.

Here is a more interesting example of a RCI algebra where formulas (2.2.9) are not trivial.

This very short proof was explained to us by G. Lusztig 
Example 5.3.3. Let $m \geq 1$, and fix an $m$-tuple $q_{1}, \ldots, q_{m} \in \mathbb{C}^{\times}$. Let $A$ be the algebra with $m$ generators $x_{i}, i=1, \ldots, m$, and one relation

$$
\operatorname{ad}_{q_{1}}\left(x_{1}\right) \operatorname{ad}_{q_{2}}\left(x_{2}\right) \ldots \operatorname{ad}_{q_{m-1}}\left(x_{m-1}\right) x_{m}=0,
$$

where $\operatorname{ad}_{q}(x) y:=x y-q y x$, and $q_{i} \in \mathbb{C}^{\times}$are not roots of 1 . We claim that it is a $\mathrm{RCI}$ algebra. Indeed, we can rewrite the relations as

$$
\begin{aligned}
x_{m-1} x_{m}-q_{m-1} x_{m} x_{m-1}=z_{m-1}, \quad & x_{m-2} z_{m-1}-q_{m-2} z_{m-1} x_{m-2}=z_{m-3}, \ldots, \\
& x_{1} z_{2}-q_{1} z_{2} x_{1}=0 .
\end{aligned}
$$

We need to show that the representation space of this algebra has dimension $n^{2}(m-1)$. We assign degree 1 to all variables, $x_{i}, z_{j}$. The corresponding top degree homogeneous terms of the above equations read

$$
\begin{aligned}
x_{m-1} x_{m}-q_{m-1} x_{m} x_{m-1}=0, \quad & x_{m-2} z_{m-1}-q_{m-2} z_{m-1} x_{m-2}=0, \ldots, \\
& x_{1} z_{2}-q_{1} z_{2} x_{1}=0 .
\end{aligned}
$$

we find that this defines a complete intersection by Proposition 5.3.1 (so the dimension of the space of solutions is the expected number $n^{2}(m-1)$ ). Therefore, the original equations also have the space of solutions of this dimension, as desired.

5.4. Examples of asymptotic RCI algebras. We begin with some combinatorial preliminaries. Let $e$ and $f$ be two (possibly equal) words in the alphabet $v_{1}, \ldots, v_{n}$. We will say that $e, f$ are non-overlapping if none of them is a proper subword of the other, and none of them begins the way the other one ends. It is clear that $e, f$ are non-overlapping if and only if any word $w$ that contains both $e$ and $f$ (in different ways if $e=f$ ) is of the form $w_{1} e w_{2} f w_{3}$ or $w_{1} f w_{2} e w_{3}$.

Let $V$ be a vector space with basis $v_{1}, \ldots, v_{n}$. We have a free $\mathbb{C}$-algebra $T V=$ $\mathbb{C}\left\langle v_{1}, \ldots, v_{n}\right\rangle$, where $v_{k}$ is assigned some grade degree $m_{k}$. Given homogeneous elements $e_{1}, \ldots, e_{p} \in T V$, we put $A=T V /(L)=\mathbb{C}\left\langle v_{1}, \ldots, v_{n}\right\rangle /\left(e_{1}, \ldots, e_{p}\right)$.

Theorem 5.4.1. Suppose that the defining relations $e_{1}, \ldots, e_{p}$ of $A$ are monomial (i.e. each is given by a single word) and pairwise non-overlapping. Then $A$ is an asymptotic RCI.

Note that the condition that $e_{1}, \ldots, e_{p}$ be pairwise non-overlapping includes the stipulation that each $e_{i}$ is non-overlapping with itself. Anick uses the name 'strongly free' for such a pairwise non-overlapping set $e_{1}, \ldots, e_{p}$, see [An].

Proof. First, one shows by a standard inclusion-exclusion argument that Anick's complex $C . A$ is a resolution of $A$. Thus, $A$ is NCCI.

Next, observe that in our case, we have $L^{\circ}=0$ (this follows from the fact that the relations are non-overlapping). Hence, by Theorem 3.7.7, we must only prove that the complex $K_{\bullet}^{\mathrm{cyc}} A$ has vanishing homology in all positive degrees. 
Remark 5.4.2. The vanishing of the homology groups of $K_{0}^{\mathrm{cyc}} A$ of degrees $>1$ is a consequence of Proposition 3.7.1, but it may also be proved directly as follows.

For brevity, write $Q_{i}=K_{i}^{\mathrm{cyc}} A, i \geq 0$. We have a natural decomposition $Q_{.}=\oplus_{w} Q_{\bullet}(w)$, where the summation is taken over cyclic words $w$, and $Q_{i}(w)$ for $i>0$ is the span of all elements $w_{1} \otimes e_{j_{1}} \otimes \ldots \otimes w_{i} \otimes e_{j_{i}}$ such that $w_{1} e_{j_{1}} \ldots w_{i} e_{j_{i}}=w$, while $Q_{0}(w)$ is a 1 -dimensional vector space spanned by $w$.

Let $w$ be a cyclic word. Since the relations are non-overlapping, the word $w$ has a unique (up to cyclic permutations) maximal length representation as $w=z_{1} \ldots z_{m}$, where $z_{i}:=w_{i} e_{j_{i}}$ (in this case, $w$ contains exactly $m$ distinct subwords of the form $e_{i}$ ). Let $D$. be the 2-step complex with $D_{0}=D_{1}=\mathbb{C}$, and $d: D_{1} \rightarrow D_{0}$ the identity map. It is easy to check that if $w$ contains at least one monomial $e_{i}$, and $\Gamma=\mathbb{Z} / n \mathbb{Z}$ is the cyclic symmetry group of $w$ (clearly, $m$ is divisible by $n)$, then the complex $Q \cdot(w)$ is isomorphic to $\left(\left(D_{\bullet}\right)^{\otimes m}\right)^{\Gamma}$ and therefore is exact. On the other hand, if $w$ does not contain $e_{i}$, then $Q .(w)$ is concentrated in degree 0 . This implies that the complex $Q$. is exact in positive degrees, as desired. Theorem 5.4.1 is proved.

Definition 5.4.3. Fix an alphabet $v_{1}, \ldots, v_{n}$ and let $\left(m_{1}, \ldots, m_{n} ; r_{1}, \ldots, r_{p}\right)$ be a collection of nonnegative degrees. For each $i=1, \ldots, n$, put $\operatorname{deg} v_{i}=m_{i}$. We say that the above collection of degrees is admissible if there exist pairwise nonoverlapping words $w_{1}, \ldots w_{p}$, in our alphabet, such that $\operatorname{deg} w_{j}=r_{j}, j=1, \ldots p$.

Let $v_{1}, \ldots, v_{n}$ be indeterminates of degrees $m_{1}, \ldots, m_{n}$. One says that a certain statement holds for a Weil generic $p$-tuple of homogeneous elements $e_{1}, . ., e_{p} \in$ $\mathbb{C}\left\langle v_{1}, \ldots, v_{n}\right\rangle$, of degrees $r_{1}, \ldots, r_{p}$ if it holds for $p$-tuples which belong to an at most countable intersection of nonempty Zariski open sets in the direct sum of the homogeneous components of the algebra $\mathbb{C}\left\langle v_{1}, \ldots, v_{n}\right\rangle$ of degrees $r_{1}, \ldots, r_{p}$.

Theorem 5.4.4. Let $\left(m_{1}, \ldots, m_{n} ; r_{1}, \ldots, r_{p}\right)$ be an admissible collection of degrees and put $\operatorname{deg} v_{j}=m_{j}, j=1, \ldots, n$. Then, for a Weil generic set of homogeneous relations $e_{1}, . ., e_{p} \in \mathbb{C}\left\langle v_{1}, \ldots, v_{n}\right\rangle$, of degrees $r_{1}, \ldots, r_{p}$ respectively, the algebra $\mathbb{C}\left\langle v_{1}, \ldots, v_{n}\right\rangle /\left(e_{1}, \ldots, e_{p}\right)$ is an asymptotic $R C I$.

Proof. For each $j \geq 0$, the property of the complex $K_{\cdot}^{\text {cyc }} A$ to have vanishing higher homology groups in degree $j$ is clearly an open condition. Thus, Theorem 5.4.4 is an immediate consequence of Theorem 5.4.1.

We refer to [An3] and [Pi, Theorem 2.25] for related results on NCCI algebras with generic relations.

Example 5.4.5. The collection $(1,1 ; r)$ is admissible for any $r>1$. Indeed, if $x, y$ are generators then the word $x^{r-1} y$ is not self-overlapping. Thus, an algebra $A$ with two generators of degree 1 and one Weil generic relation of degree $r$ is an 
asymptotic RCI, by Theorem 5.4.4. However, such an algebra is not RCI for any $r \geq 4$. Indeed, in this case there are 4-dimensional representations where the generators $x, y$ act by any strictly upper triangular matrices in some common basis. The dimension of the set of such representations is 18 . Thus the dimension of the space of $N \geq 4$ dimensional representations which are isomorphic to the sum of one of the above 4-dimensional representations and a bunch of 1-dimensional representations is $N^{2}+2$, which is bigger than $N^{2}$, required by the RCI property.

Now consider collections $(1,1 ; r, s), r, s>1$. If $r=2,3$, then it is easy to see that such a collection is never admissible. If $r=4$, then the smallest $s$ for which this collection is admissible is $s=5$; the corresponding words are $x^{2} y^{2}$ and $x y x y^{2}$. For randomly chosen relations of degrees $(1,1 ; 4,4)$, the corresponding algebra is not NCCI.

\section{Preprojective algebras and Quiver varieties}

6.1. Hilbert series for preprojective algebras. An important example, which was the main motivation for this study, is that of preprojective algebras.

Recall the setting of $\S 1.3$. Thus, $\Pi$ is the preprojective algebra of a connected quiver $Q$ with vertex set $I$. We have $\Pi=T_{R} V /(L)$, where $V$ is a $\mathbb{C}$-vector space with basis formed by the edges of the quiver $\bar{Q}$, and $L$ is an $R$-subbimodule in $V \otimes_{R} V$ generated by the element $\sum_{a \in Q}\left[a, a^{*}\right]$. Therefore, we get $h(V ; t)=t \cdot \mathbf{c}$, and $h(L ; t)=t^{2} \cdot \mathbf{1}$. In this case, one can rewrite the $\zeta$-function in $(2.2 .4)$ in terms of the adjacency matrix $\mathbf{c}$ of the double $\bar{Q}$, as follows

$$
\zeta(Q ; t):=\zeta(V, L ; t)=\prod_{s \geq 1} \frac{1}{\operatorname{det}\left(\mathbf{1}-t^{s} \cdot \mathbf{c}+t^{2 s} \cdot \mathbf{1}\right)}
$$

The vector space $L^{\circ}$ is spanned by a single degree 2 element $\sum_{a \in Q}\left[a, a^{*}\right]$, hence, we have $\lambda\left(L^{\circ}\right)=1-t^{2}$.

According to $[\mathrm{CB}]$ (see explanations in [CBEG], section 11), we know that $\Pi$ is an RCI algebra, provided the quiver $Q$ is neither Dynkin nor extended Dynkin. Thus, Theorem 1.3.1 follows from Theorem 2.2.7.

Example 6.1.2. Fix an integer $g \geq 1$. For any $n \geq 1$, let $A_{g, n}$ be the algebra with generators $x_{i}, y_{i}, i=1, \ldots, g$, and one defining relation $\left(\sum\left[x_{i}, y_{i}\right]\right)^{n}=0$.

In the special case $n=1$, the corresponding algebra $A_{g, 1}$ is nothing but the preprojective algebra of a quiver with one vertex and $g$ edge-loops. This preprojective algebra may be thought of as an 'additive' (i.e. Lie algebra) analogue of the group algebra of the fundamental group of a genus $g$ Riemann surface. 
Proposition 6.1.3. Let $g>1$. Then, for $n=1$, we have

$$
h\left(A_{g, 1}\right)=\frac{1}{1-2 g \cdot t+t^{2}}, \quad \text { and } \quad h(\mathcal{O}(\Pi))=\frac{1}{1-t^{2}} \prod_{s=1}^{\infty} \frac{1}{1-2 g \cdot t^{s}+t^{2 s}} .
$$

For any $n \geq 2$, we have

$$
\begin{aligned}
h\left(A_{g, n}\right) & =\frac{1-t^{2 n}}{1-2 g t+2 g t^{2 n+1}-t^{2 n+2}}, \\
h\left(\mathcal{O}\left(A_{g, n}\right)\right) & =\prod_{s=1}^{\infty} \frac{1-t^{2(n-1+s)}}{\left.1-2 g t^{s}+2 g t^{(2 n+1) s}-t^{(2 n+2) s}\right)} .
\end{aligned}
$$

Proof. In the case $n=1$, the formulas follow from Theorem 1.3.1.

If $n \geq 2$, we apply the results of $\S 5.1$. Specifically, in the notation of that section, we take $B=A_{g, 1}$ and $D=\mathbb{C}[z] /\left(z^{n}\right)$, and also $Q=0$. Thus, one has

$$
h(D)=\frac{1-t^{2 n}}{1-t^{2}}, \quad \text { and } \quad h(\mathcal{O}(D))=\prod_{i=1}^{n-1} \frac{1}{1-t^{2 i}} .
$$

The algebra $A_{g, 1}$ is an asymptotic RCI and we have $A_{g, n}=A_{g, 1}{ }^{\circ} D$. Thus, from the formulas for $n=1$, applying Proposition 5.1.6 and Proposition 5.2.1, we get the required formulas for $n>1$.

We note that the formula of Proposition 6.1.3 for $h\left(\mathcal{O}\left(A_{g, 1}\right)\right)$ fails if $g=1$. Theorem 1.3.1 cannot be applied to that case, since the quiver with one vertex and one edge-loop is an affine Dynkin quiver. In this case, the representation scheme $\operatorname{Rep}_{d}\left(A_{g, 1}\right)$ is the commuting scheme, consisting of pairs of matrices $X, Y$ of size $d$ such that $X Y-Y X=0$. This scheme has dimension $d^{2}+d$ and is not a complete intersection unless $d=1$.

Note also that, for $n>1$, the algebra $A_{g, n}$ (as well as the algebra $D=$ $\left.\mathbb{C}[z] /\left(z^{n}\right)\right)$ is not NCCI and it has infinite cohomological dimension.

Next, let $Q$ be an arbitrary quiver and let $J \subset I$ be a subset of the set of vertices of $Q$. Following [EE], define the partial preprojective algebra $A=\Pi_{Q, J}$ in the same way as $\Pi_{Q}$ except that the defining relation has the form

$$
\left(1-p_{J}\right) \sum\left[a, a^{*}\right]\left(1-p_{J}\right)=0,
$$

where $p_{J}$ is the sum of the idempotents corresponding to the vertices from $J$.

Proposition 6.1.4. If $J \neq \emptyset$ then, for any connected quiver $Q$, the partial preprojective algebra $\Pi_{Q, J}$ is $R C I$. 
Proof. If $Q$ has only one vertex, the statement is clear, so we will assume that $Q$ has more than one vertex. Let $j \in J$, and let $Q^{\prime}$ be the quiver $Q$ with an additional self-loop $\gamma$ at the vertex $j$. Then $\Pi_{Q^{\prime}, J}=\Pi_{Q, J} *_{R} \Pi_{\gamma, J}$, where $\gamma$ stands for the quiver with vertex set $I$ and unique edge $\gamma$. The quiver $Q^{\prime}$ is not Dynkin or affine Dynkin. Therefore, by Theorem 1.3.1, $\Pi_{Q^{\prime}}$ is a RCI algebra, and hence by Lemma 5.1.2, (i), $\Pi_{Q^{\prime}, J}$ is a RCI algebra. Hence by Lemma 5.1.2, (ii), so is $\Pi_{Q, J}$, as desired.

Thus we obtain the following result.

Proposition 6.1.5. Let $Q$ be a connected quiver with vertex set $I$ and let $J \subset I$ be a nonempty subset. Let $\mathbf{c}$ be the adjacency matrix of $\bar{Q}$ and $\mathbf{1}_{I \backslash J}$ be the diagonal matrix whose principal diagonal is the characteristic function of the set $I \backslash J$. Then, we have

$h\left(\Pi_{Q, J}\right)=\left(\mathbf{1}-\mathbf{c} t+t^{2} \cdot \mathbf{1}_{I \backslash J}\right)^{-1}, \quad$ and $\quad h\left(\mathcal{O}\left(\Pi_{Q, J}\right)\right)=\prod_{s=1}^{\infty} \frac{1}{\operatorname{det}\left(\mathbf{1}-t^{s} \cdot \mathbf{c}+t^{2 s} \cdot \mathbf{1}_{I \backslash J}\right)}$.

Remark 6.1.6. (i) The formula for $h\left(\Pi_{Q, J}\right)$ was proved in [EE] by another method.

(ii) Note that there is no factor $\left(1-t^{2}\right)^{-1}$ in the formula for $h\left(\mathcal{O}\left(\Pi_{Q, J}\right)\right)$ since $L^{\circ}=0$ in this case.

6.2. Asymptotic Hilbert series for Nakajima quiver varieties. Fix a pair of dimension vectors $\mathbf{d}=\left\{d_{i}\right\}_{i \in I}$ and $\mathbf{w}=\left\{w_{i}\right\}_{i \in I}$. For each $i \in I$, we put

$\mathbf{P}_{i}=\operatorname{Hom}\left(\mathbb{C}^{d_{i}}, \mathbb{C}^{w_{i}}\right), \quad \mathbf{P}_{\mathbf{d}, \mathbf{w}}=\bigoplus_{i \in I} \mathbf{P}_{i}, \quad \mathbf{Q}_{i}=\operatorname{Hom}\left(\mathbb{C}^{w_{i}}, \mathbb{C}^{d_{i}}\right), \quad \mathbf{Q}_{\mathbf{w}, \mathbf{d}}=\bigoplus_{i \in I} \mathbf{Q}_{i}$

We write elements of $\mathbf{P}$, resp. of $\mathbf{Q}$, as $\mathbf{p}=\left\{p_{i} \in \mathbf{P}_{i}\right\}$, resp. $\mathbf{q}=\left\{q_{i} \in \mathbf{Q}_{i}\right\}$. Thus, the composite $q_{i} \circ p_{i}$ is a linear map $\mathbb{C}^{d_{i}} \rightarrow \mathbb{C}^{d_{i}}$, that is, an element of $\mathbf{E}_{i i}=\operatorname{Hom}\left(\mathbb{C}^{d_{i}}, \mathbb{C}^{d_{i}}\right)$.

Now, let $Q$ be a connected quiver. For any edge $a \in \bar{Q}$, we put $\epsilon_{a}:=1$ if $a \in Q$, resp. $\epsilon_{a}:=-1$ if $a \in \bar{Q} \backslash Q$. Let $V$ be an $R$-bimodule with basis formed by the edges of $\bar{Q}$. We have $\operatorname{Rep}_{\mathbf{d}}(\bar{Q})=\operatorname{Hom}_{R \text {-bimod }}\left(V, \mathbf{E}_{\mathbf{d}}\right)=\mathbb{V}_{\mathbf{d}}$.

Nakajima considers a moment map

$$
\begin{array}{r}
\mu_{\mathbf{d}, \mathbf{w}}: \mathbb{V}_{\mathbf{d}} \times \mathbf{P}_{\mathbf{d}, \mathbf{w}} \times \mathbf{Q}_{\mathbf{w}, \mathbf{d}} \rightarrow \oplus_{i \in I} \mathbf{E}_{i i}, \quad(\rho, \mathbf{p}, \mathbf{q}) \mapsto \oplus_{i \in I} \mu_{i}(\rho, \mathbf{p}, \mathbf{q}), \\
\mu_{i}(\rho, \mathbf{p}, \mathbf{q}):=\left(\sum_{\{a \in \bar{Q} \mid \operatorname{tail}(a)=i\}} \epsilon_{a} \cdot \rho\left(a^{*}\right) \cdot \rho(a)\right)-q_{i} \circ p_{i} \in \mathbf{E}_{i i}, \\
\forall i \in I, \rho \in \operatorname{Rep}_{\mathbf{d}}(\bar{Q}) .
\end{array}
$$


The affine quiver variety with parameters $\mathbf{d}$ and $\mathbf{w}$ is defined as a categorical quotient $\mathfrak{M}(\mathbf{d}, \mathbf{w}):=\mu_{\mathbf{d}, \mathbf{w}}^{-1}(0) / / G_{\mathbf{d}}$. There is an action of the group $G_{\mathbf{w}}=$ $\prod G L\left(\mathbb{C}^{w_{i}}\right)$ on $\mathfrak{M}(\mathbf{d}, \mathbf{w})$ induced by the natural $G_{\mathbf{w}}$-action on $\mathbb{C}^{\mathbf{w}}$. There is also a $\mathbb{C}^{\times}$-action on $\mathfrak{M}(\mathbf{d}, \mathbf{w})$ arising from the standard $\mathbb{C}^{\times}$-action on the vector space $\mathbb{V}_{\mathbf{d}} \times \mathbf{P}_{\mathbf{d}, \mathbf{w}} \times \mathbf{Q}_{\mathbf{w}, \mathbf{d}}$, by dilation. Thus, we get a weight grading $\mathbb{C}[\mathfrak{M}(\mathbf{d}, \mathbf{w})]=$ $\bigoplus_{r \geq 0} \mathbb{C}[\mathfrak{M}(\mathbf{d}, \mathbf{w})][r]$, such that, for each $r \geq 0$, the weight component $\mathbb{C}[\mathfrak{M}(\mathbf{d}, \mathbf{w})][r]$ is a finite dimensional vector space equipped with $G_{\mathbf{w}}$-action.

For any element $\mathbf{g}=\left(g_{1}, \ldots, g_{r}\right) \in G_{\mathbf{w}}=\prod G L\left(\mathbb{C}^{w_{i}}\right)$ we form a generating function

$$
\Phi(\mathbb{C}[\mathfrak{M}(\mathbf{d}, \mathbf{w})] ; \mathbf{g}, t)=\sum_{r \geq 0} t^{r} \cdot \operatorname{Tr}\left(\left.\mathbf{g}\right|_{\mathbb{C}[\mathfrak{M}(\mathbf{d}, \mathbf{w})][r]}\right) \in \mathbb{C}[[t]] .
$$

Further, for each pair $i, j \in I$, we define $\mathbf{W}_{i j}=\operatorname{Hom}\left(\mathbb{C}^{w_{i}}, \mathbb{C}^{w_{j}}\right)=\left(\mathbb{C}^{w_{i}}\right)^{*} \otimes \mathbb{C}^{w_{j}}$. Given $g_{i} \in G L\left(w_{i}\right)$ and $g_{j} \in G L\left(w_{j}\right)$, we have a map $g_{i j}=g_{i}^{\vee} \otimes g_{j}:\left(\mathbb{C}^{w_{i}}\right)^{*} \otimes \mathbb{C}^{w_{j}} \rightarrow$ $\left(\mathbb{C}^{w_{i}}\right)^{*} \otimes \mathbb{C}^{w_{j}}$. Thus, $g_{i j} \in$ End $\mathbf{W}_{i j}$, and we get a sequence of polynomials

$$
\operatorname{det}\left(\mathbf{1}_{\mathbf{W}_{i j}}-t^{m} \cdot g_{i j}\right) \in \mathbb{C}[t], \quad m=0,1, \ldots
$$

Let $\phi_{k} \in \mathbb{C}[x], k=0,1, \ldots$, be a sequence of polynomials (essentially, Chebyshev polynomials of the second kind) in an indeterminate $x$ defined as the coefficients in the expansion of the generating function

$$
\sum_{k=0}^{\infty} \phi_{k}(x) t^{k}=\frac{1}{1-t x+t^{2}}
$$

We set $\phi_{k}=0$ for $k$ negative. Then, for each $k \in \mathbb{Z}$, we have an $I \times I$-matrix $\left\|\phi(\mathbf{c})_{i j}\right\|$. Further, recall the notation $\zeta(Q, t)$ from (6.1.1).

An argument similar to that used in the proof of [CBEG], Lemma 11.1.2, implies that, for any non-Dynkin quiver and any given dimension vector $\mathbf{w} \neq 0$, there exists a sequence of dimension vectors $\mathbf{d} \rightarrow \infty$ such that the corresponding varieties $\mu_{\mathbf{d}, \mathbf{w}}^{-1}(0)$ are all complete intersections. Thus, repeating the arguments of $\S 2.3$ one obtains, via a matrix integral calculation similar to one carried out in $\S 2.4$, the following version of Proposition 2.4.9 for quiver varieties.

Proposition 6.2.2. Fix a connected non-Dynkin quiver $Q$ and a dimension vector $\mathbf{w} \neq 0$. For any $\mathbf{g} \in G_{\mathbf{w}}$ and $r \geq 0$, the sequence of $r$-th coefficients in the formal power series $\Phi(\mathbb{C}[\mathfrak{M}(\mathbf{d}, \mathbf{w})])$ stabilizes as $\mathbf{d} \rightarrow \infty$, and for the corresponding limit we have

$$
\lim _{\mathbf{d} \rightarrow \infty} \Phi(\mathbb{C}[\mathfrak{M}(\mathbf{d}, \mathbf{w})] ; \mathbf{g}, t)=\zeta(Q, t) \cdot \prod_{i, j} \prod_{k \geq 0} \frac{1}{\operatorname{det}\left(\mathbf{1}_{\mathbf{W}_{i j}}-t^{k+2} \cdot g_{i j}\right)^{\phi_{k}(\mathbf{c})_{i j}}} .
$$

Next, we set $\left\langle\mathbf{w}, \phi_{k}(\mathbf{c}) \mathbf{w}\right\rangle:=\sum_{i, j \in I} \phi_{k}(\mathbf{c})_{i j} \cdot w_{i} \cdot w_{j}$. Taking $\mathbf{g}=1$ in the formula of the above Proposition, we deduce 
Corollary 6.2.3. With the notation and assumptions of Proposition 6.2.2, for Hilbert series of quiver varieties $\mathfrak{M}(\mathbf{d}, \mathbf{w})$ we have

$\lim _{\mathbf{d} \rightarrow \infty} h(\mathbb{C}[\mathfrak{M}(\mathbf{d}, \mathbf{w})] ; t)=\prod_{s \geq 1} \frac{1}{\operatorname{det}\left(\mathbf{1}-t^{s} \cdot \mathbf{c}+t^{2 s} \cdot \mathbf{1}\right)} \cdot \prod_{k \geq 0} \frac{1}{\left(1-t^{k+2}\right)^{\left\langle\mathbf{w}, \phi_{k}(\mathbf{c}) \mathbf{w}\right\rangle}}$

Remark 6.2.4. It was kindly pointed out to us by H. Nakajima that, in [NY], the authors study a function similar to $\Phi(\mathbb{C}[\mathfrak{M}(\mathbf{d}, \mathbf{w})] ; \mathbf{g}, t)$. The problem solved in $[N Y]$ is, however, quite different. Specifically, the setup of $[N Y]$ corresponds to the special case where $Q$ is the Jordan quiver. In that special case, Nakajima and Yoshioka analyze asymptotic behavior with respect to the variable $t$ (corresponding, in the notation of $[\mathrm{NY}]$, to $e^{\varepsilon_{1}+\varepsilon_{2}}$ ) for fixed dimension vector $\mathbf{d}$. The resulting function (for finite $\mathbf{d}$ ) is much more complicated than the one given in Proposition 6.2.2; it is a $K$-theoretic version of the Seiberg-Witten prepotential, see $[\mathrm{NY}]$ for details.

Remark 6.2.5. Fix a total ordering on $I$, the set of vertices of $Q$, and put $G S_{Q}:=$ $\prod_{i<j} G L\left(\mathbf{c}_{i j}\right) \times \prod_{i \in I} S p\left(\mathbf{c}_{i i}\right)$. The group $G S_{Q}$ acts naturally on the vector space spanned by the edges of $\bar{Q}$ by linear transformations. This gives a $G S_{Q^{-}}$action on the path algebra of $\bar{Q}$ and on the algebra $\Pi(Q)$, by algebra automorphisms. Therefore, for any pair $(\mathbf{d}, \mathbf{w})$, of dimension vectors, one has a linear $G S_{Q}$-action on the symplectic vector space $\mathbb{V}_{\mathbf{d}} \times \mathbf{P}_{\mathbf{d}, \mathbf{w}} \times \mathbf{Q}_{\mathbf{w}, \mathbf{d}}$ by symplectic automorphisms. This action commutes both with the $G_{\mathbf{d}^{-}}$action and $G_{\mathbf{w}}$-action, as well as with the $\mathbb{C}^{\times}$-action. Thus, the quiver variety $\mathfrak{M}(\mathbf{d}, \mathbf{w})$ acquires the structure of a $G_{\mathrm{w}} \times \mathbb{C}^{\times} \times G S_{Q^{-v a r i e t y} \text {. }}$

Now, let $\mathrm{R}\left(G S_{Q}\right)$ denote the representation ring of finite dimensional rational $G S_{Q}$-modules. One can introduce $G S_{Q}$-equivariant refinements of the formal series $h(\Pi ; t), \Phi(\mathbb{C}[\mathfrak{M}(\mathbf{d}, \mathbf{w})] ; \mathbf{g}, t)$, etc., as appropriately defined elements of $\mathrm{R}\left(G S_{Q}\right)[[t]]$, the ring of formal power series with $\mathrm{R}\left(G S_{Q}\right)$-coefficients. The formulas of Theorem 1.3.1 and of Proposition 6.2.2 still apply in this refined $G S_{Q^{-}}$ equivariant framework, provided the symbol $\mathbf{c}_{i j}$ is understood as an element of $\mathrm{R}\left(G S_{Q}\right)$ corresponding to the class of the fundamental vector representation of the group $G L\left(\mathbf{c}_{i j}\right)$ if $i<j$, resp., to the class of the fundamental covector representation of the group $G L\left(\mathbf{c}_{j i}\right)$ if $i>j$, and also as the class of the vector representation of the group $S p\left(\mathbf{c}_{i i}\right)$ if $i=j$ (this way, $\mathbf{c}$ becomes an $\mathrm{R}\left(G S_{Q}\right.$ )valued matrix).

6.3. The case of extended Dynkin quivers. Let $Q$ be an extended Dynkin quiver, and write $o \in I$ for an extending vertex. We let $e_{o}$ denote the idempotent in the preprojective algebra of $Q$ corresponding to the trivial path at $O$. 
Let $\Gamma \subset S L_{2}(\mathbb{C})$ be the finite subgroup corresponding to $Q$ via the McKay correspondence. Thus, the extending vertex corresponds to the trivial representation of $\Gamma$. We write $M$ for the 2-dimensional tautological representation of $\Gamma$ and let $\Gamma \ltimes \operatorname{Sym} M$ be the corresponding smash-product algebra.

According to $[\mathrm{CBH}]$, one has

Proposition 6.3.1. (i) The preprojective algebra $\Pi$ is Morita equivalent to $\Gamma \ltimes$ Sym $M$.

(ii) There is a graded algebra isomorphism $(\operatorname{Sym} M)^{\Gamma} \cong e_{o} \Pi e_{o}$.

Part (i) of the Proposition implies, in particular, that Anick's complex is a resolution for the preprojective algebra $\Pi$, hence, $\Pi$ is NCCI. Therefore, Theorem 3.2.4(2) and the second formula in (3.7.3) yield

$$
h(\Pi)=\left(\mathbf{1}-t \cdot \mathbf{c}+t^{2} \cdot \mathbf{1}\right)^{-1}, \quad \text { resp. }, \quad h(\mathcal{O}(\Pi))=\zeta(Q, t) \cdot h\left(\operatorname{Sym} H H_{2}(\Pi)\right) .
$$

Recall Chebyshev polynomials of the first kind, $\varphi_{k}$, introduced in (1.3.2), and Chebyshev polynomials of the second kind, $\phi_{k}$, introduced in (6.2.1). These polynomials are related by the formula $\varphi_{k}=\phi_{k}-\phi_{k-2}, k=0,1, \ldots$

We have the following well known result, cf. [Su].

Corollary 6.3.3. The Hilbert series of the graded algebra $(\operatorname{Sym} M)^{\Gamma}$ is given by the formula

$$
h\left((\operatorname{Sym} M)^{\Gamma}\right)=\sum_{k \geq 0} \phi_{k}(\mathbf{c})_{o o} \cdot t^{k} .
$$

Proof. We use the first formula in (6.3.2), which is a matrix equation. Hence, for the matrix element on each side of that equation corresponding to the extending vertex, we get

$$
h\left(e_{o} \Pi e_{o}\right)=h(\Pi)_{o o}=\left(\left(\mathbf{1}-t \cdot \mathbf{c}+t^{2} \mathbf{1}\right)^{-1}\right)_{o o}=\sum_{k \geq 0} \phi_{k}(\mathbf{c})_{o o} \cdot t^{k},
$$

where the last equality is the definition of the polynomials $\phi_{k}$ given in (6.2.1). The result now follows from the isomorphism of Proposition 6.3.1(ii).

Proof of identity (1.3.3). We will be interested in Hochschild cohomology of the smash-product $\Gamma \ltimes \operatorname{Sym} M$. These are well known and easy to find. To describe the answer, put $\mathscr{S}:=\Gamma \backslash\{1\}$, and let $\mathbb{C}[\mathscr{S}]^{\Gamma}$ denote the vector space of class functions on the finite set $\mathscr{S}$. Then, one has, see [CBEG], formula (8.5.1):

$$
H H^{i}(\Gamma \ltimes \operatorname{Sym} M)= \begin{cases}(\operatorname{Sym} M)^{\Gamma} & \text { if } i=0 \\ \left(M^{*} \otimes \operatorname{Sym} M\right)^{\Gamma} & \text { if } i=1 \\ \Lambda^{2} M^{*} \otimes(\mathbb{C}[\mathscr{S}] \oplus \operatorname{Sym} M)^{\Gamma} & \text { if } i=2 \\ 0 & \text { if } i>2 .\end{cases}
$$


It follows by Morita equivalence of Hochschild homology and Van den Bergh duality $\Lambda^{2} M^{*} \otimes H H .(\Pi) \cong H H^{2-\cdot}(\Pi)$, see $[\mathrm{VdB}]$, that we have

$$
\begin{gathered}
(6.3 .4) \Lambda^{2} M^{*} \otimes H H_{2}(\Pi) \cong H H^{0}(\Pi) \cong H H^{0}(\Gamma \ltimes \operatorname{Sym} M) \cong(\operatorname{Sym} M)^{\Gamma}, \\
\Lambda^{2} M^{*} \otimes H H_{0}(\Pi) \cong H H^{2}(\Pi) \cong H H^{2}(\Gamma \ltimes \operatorname{Sym} M) \cong \Lambda^{2} M^{*} \otimes(\mathbb{C}[\mathscr{S}] \oplus \operatorname{Sym} M)^{\Gamma} .
\end{gathered}
$$

The isomorphisms in the second line of (6.3.4) imply that $\left(H H_{0}(\Pi)\right)_{+} \cong$ $(\operatorname{Sym} M)_{+}^{\Gamma}$. Hence, in terms of the integer coefficients $\phi_{k}(\mathbf{c})_{o o}$ in the Hilbert series of $(\operatorname{Sym} M)^{\Gamma}$, see Corollary 6.3.3, we obtain the formula

$$
h(\mathcal{O}(\Pi))=h\left(\operatorname{Sym}\left(H H_{0}(\Pi)_{+}\right)\right)=h\left(\operatorname{Sym}\left((\operatorname{Sym} M)_{+}^{\Gamma}\right)\right)=\prod_{k \geq 1} \frac{1}{\left(1-t^{k}\right)^{\phi_{k}(\mathbf{c})_{o o}}} .
$$

Further, the vector space $\Lambda^{2} M$ has weight degree 2 . Hence, from the first line of (6.3.4) we get a graded space isomorphism $H_{2}(\Pi) \cong(\operatorname{Sym} M)^{\Gamma}\langle 2\rangle$, where $\langle 2\rangle$ denotes grading shift by 2 . Thus, we find

$$
h\left(\operatorname{Sym} H H_{2}(\Pi)\right)=h\left(\operatorname{Sym}\left((\operatorname{Sym} M)^{\Gamma}\langle 2\rangle\right)\right)=\prod_{k \geq 0} \frac{1}{\left(1-t^{k+2}\right)^{\phi_{k}(\mathbf{c})_{o o}}} .
$$

Using the above formulas for Hilbert series, the last equation can be rewritten as

$$
\prod_{k \geq 1} \frac{1}{\left(1-t^{k}\right)^{\phi_{k}(\mathbf{c})_{o o}}}=\zeta(Q, t) \cdot \prod_{k \geq 0} \frac{1}{\left(1-t^{k+2}\right)^{\phi_{k}(\mathbf{c})_{o o}}} .
$$

Thus, we obtain

$$
\zeta(Q, t)=\prod_{k \geq 1} \frac{1}{\left(1-t^{k}\right)^{\phi_{k}(c)_{00}-\phi_{k-2}(c)_{00}}}=\prod_{k \geq 1} \frac{1}{\left(1-t^{k}\right)^{\varphi_{k}(\mathbf{c})_{o o}} .}
$$

This equation is equivalent to (1.3.3), and we are done.

Remark 6.3.5. The identity in (1.3.3) may be also verified directly, case by case, for each Dynkin diagram of type $A, D, E$. To do so, one has to know explicit formulas for the determinant of the $t$-analogue of the Cartan matrix of the corresponding extended Dynkin graph. Equivalently, for the polynomial $D(t):=\operatorname{det}\left(\mathbf{1}-t \cdot \mathbf{c}+t^{2} \cdot \mathbf{1}\right)$, one finds the following formulas, see [Lu, $\left.\S 3.13\right]$ :

$$
\begin{aligned}
& A_{n}: D(t)=\left(1-t^{n+1}\right)^{2} \\
& D_{n}: D(t)=\left(1-t^{4}\right)^{2}\left(1-t^{2 n-4}\right) /\left(1-t^{2}\right) \\
& E_{6}: D(t)=\left(1-t^{4}\right)\left(1-t^{6}\right)^{2} /\left(1-t^{2}\right) \\
& E_{7}: D(t)=\left(1-t^{4}\right)\left(1-t^{6}\right)\left(1-t^{8}\right) /\left(1-t^{2}\right) \\
& E_{8}: D(t)=\left(1-t^{4}\right)\left(1-t^{6}\right)\left(1-t^{10}\right) /\left(1-t^{2}\right) .
\end{aligned}
$$




\section{REFERENCES}

[AH] J.F. Adams, P.J. Hilton, On the chain algebra of a loop space. Comment. Math. Helv. 30 (1956), 305-330.

[An] D. J. Anick, Noncommutative graded algebras and their Hilbert series. J. Algebra $\mathbf{7 8}$ (1982), 120-140.

[An2] On the homology of associative algebras. Trans. Amer. Math. Soc. 296 (1986), 641-659.

[An3] Generic algebras and $C W$ complexes. Algebraic topology and algebraic $K$ theory. 247-321, Ann. of Math. Stud., 113, Princeton Univ. Press, Princeton, NJ, 1987.

[CB] W. Crawley-Boevey, Geometry of the moment map for representations of quivers. Compositio Math. 126 (2001), 257-293.

[CBEG] W. Crawley-Boevey, P. Etingof, V. Ginzburg, Noncommutative Geometry and Quiver algebras. Adv. in Math. 209 (2007), 274-336. arXiv:math.AG/0502301.

[CBH] W. Crawley-Boevey, M. Holland, Noncommutative deformations of Kleinian singularities. Duke Math. J. 92 (1998), 605-635.

[CQ] J. Cuntz, D. Quillen, Algebra extensions and nonsingularity. J. Amer. Math. Soc. 8 (1995), 251- 289.

[DS] P. Diaconis, M. Shahshahani, On the eigenvalues of random matrices. Studies in applied probability. J. Appl. Probab. 31A (1994), 49-62.

[EE] P. Etingof, C.-H. Eu, Koszulity and the Hilbert series of preprojective algebras, arXiv:math.RA/0512287.

[EG] - V. Ginzburg, Symplectic reflection algebras, Calogero-Moser system, and deformed Harish-Chandra homomorphism, Invent. Math. 147 (2002), 243-348, arXiv:math. AG/0011114.

[Gi1] V. Ginzburg, Non-commutative symplectic geometry, quiver varieties, and operads. Math. Res. Lett. 8 (2001), 377-400.

[Gi2] Double derivations and Cyclic homology. arXiv:math.KT/0505236.

[Gi3] Calabi-Yau algebras. arXiv:math. AG/0612139.

[Go] E. S. Golod, Homology of the Shafarevich complex, and noncommutative complete intersections. (Russian) Fundam. Prikl. Mat. 5 (1999), no. 1, 85-95, 97-100.

[GS] I.R. Shafarevich, On the class field tower. Izv. Akad. Nauk SSSR 28 (1964), 261-272.

[LBP] L. Le Bruyn, C. Procesi, Semisimple representations of quivers. Trans. Amer. Math. Soc. 317 (1990), 585-598.

[Lo] J.L. Loday, Cyclic homology. Grundlehren der Mathematischen Wissenschaften, 301. Springer-Verlag, Berlin, 1998.

[Lu] G. Lusztig, Some examples of square integrable representations of semisimple p-adic groups, Trans. Amer. Math. Soc. 277 (1983), 623-653.

[MOV] A. Malkin, V. Ostrik, M. Vybornov, Quiver varieties and Lusztig's algebra. Adv. Math. 203 (2006), 514-536. arXiv:math.RT/0403222.

[NY] H. Nakajima, K. Yoshioka, Instanton counting on blowup. II. K-theoretic partition function. Transform. Groups 10 (2005), 489-519. arXiv:math. AG/0505553.

[Pi] D. Piontkovsky, Extensions of differential graded algebras. J. Math. Sci. (to appear).

[Pr] C. Procesi, A formal inverse to the Cayley-Hamilton theorem. J. Algebra 107 (1987), 63-74.

[St] R. Stanley, Enumerative combinatorics, v.1, Cambridge Studies in Advanced mathematics 49, Cambridge University Press, 2002. 
[Su] R. Suter, Quantum affine Cartan matrices, Poincare series of binary polyhedral groups, and reflection representations. Manuscripta Mathem. (2007). arXiv:math. RT/0503542.

[VdB] M. Van den Bergh, A relation between Hochschild homology and cohomology for Gorenstein rings. Proc. Amer. Math. Soc. 126 (1998), 1345-1348; 130 (2002), 2809-2810.

Pavel Etingof

Department of Mathematics

Massachusetts Institute of Technology

Cambridge, MA 02139, USA

E-mail: etingof@math.mit.edu.

Victor Ginzburg

Department of Mathematics

University of Chicago

Chicago, IL 60637, USA

E-mail: ginzburg@math.uchicago.edu 